\title{
Studies into exfoliation and coating of Egyptian blue for application to the detection of latent fingermarks
}

Sorour Shahbazi ${ }^{a}$, John V. Goodpaster ${ }^{\mathrm{b}}$, Gregory D. Smith ${ }^{\mathrm{c}}$, Thomas Becker ${ }^{\mathrm{a}}$, Simon W. Lewis ${ }^{\mathrm{a},}$

${ }^{a}$ Curtin Institute of Functional Molecules and Interfaces and Department of Chemistry, Curtin University, GPO Box U1987, Perth, Western Australia 6845, Australia

${ }^{b}$ Department of Chemistry and Chemical Biology, Forensic and Investigative Sciences Program, Indiana

University Purdue University Indianapolis (IUPUI), 402 North Blackford Street, LD 326, Indianapolis, IN, 46202, USA

'Indianapolis Museum of Art at Newfields, 4000 Michigan Road, Indianapolis, IN, 46208, USA

*Corresponding author (E-mail: s.lewis@curtin.edu.au)

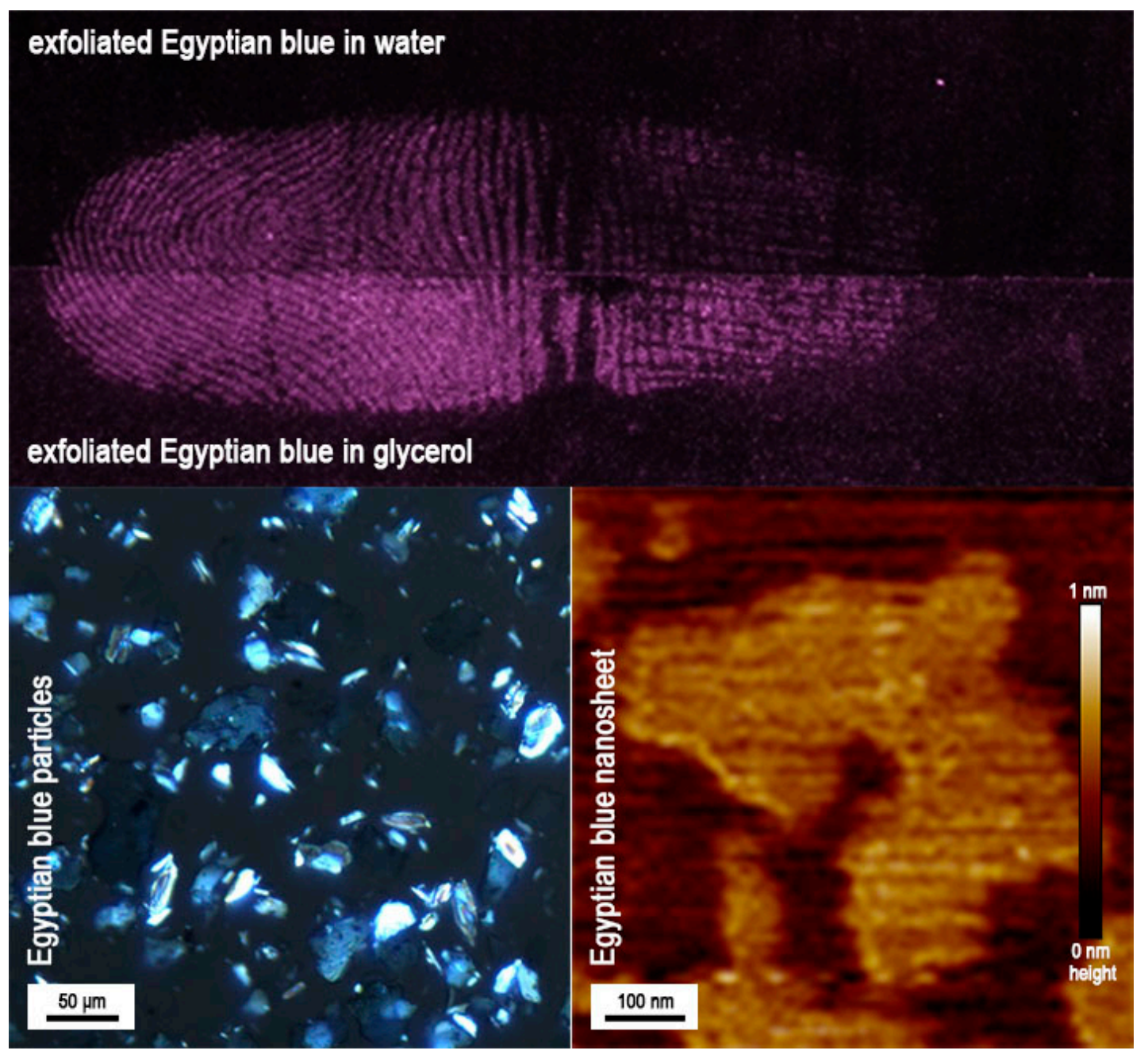




\subsection{Introduction}

We have recently demonstrated that exfoliated Egyptian blue powder coated with cetrimonium bromide is effective for detecting latent fingermarks on a range of highly-patterned non-porous surfaces. ${ }^{1}$ In this extension of that work, we here present our preliminary studies into alternative approaches to preparing exfoliated Egyptian blue with a variety of different coatings. The various powders were then applied to the detection of latent fingermarks on non-porous surfaces. This proof of concept study demonstrated that there is potential improvement in performance compared to our previous work, however more comprehensive studies are required to compare the quality of the fingermarks developed with these powders against particles exfoliated in water.

\subsection{Exfoliation of Egyptian blue powder in glycerol}

\subsection{Experiment}

Three grams of Egyptian blue (EB) powder (Kremer 10600) was vigorously stirred in $90 \mathrm{~mL}$ of glycerol $\left(\mathrm{C}_{3} \mathrm{H}_{8} \mathrm{O}_{3}, \geq 99 \%\right.$, Chem-Supply) in a round-bottom flask using a glass magnetic stir at 250 ${ }^{\circ} \mathrm{C}$ for 3 and 9 days. A block heater was used for stirring and heating and a water circulating system was used for condensation. After 4 days, the solution started boiling and after 9 days bubbles got to the condenser. Then the mixture was left to cool to room temperature. For purification, warm water was added to the mixture (approximately $45^{\circ} \mathrm{C}, 1: 3$ water:slurry ratio) and then centrifuged to separate the powder $(3000 \mathrm{rpm}$ for $3 \mathrm{~min}$ ). The powder was washed three times with warm water and then moved to a petri dish and left in the oven $\left(30^{\circ} \mathrm{C}\right)$ to dry overnight. The 3 -day and 9-day exfoliated EB in glycerol (EEB-G-3D and EEB-G-9D, respectively) were used for further experiments.

\subsection{Results and discussion}

\subsubsection{Preparation and characterisation}

There are three important parameters which affect the exfoliation process of layered structures using the liquid-phase exfoliation method: polarity of solvent, temperature, and ionic surfactant. ${ }^{2,3}$ In this technique, forces between the liquid and sheet silicate layers should overcome intermolecular forces between adjacent sheets. Also, stabilizing the sheets with suitable surfactants should prevent reaggregation. To enhance the exfoliation process, one approach is to use a polar solvent that has a higher boiling point than water to facilitate increasing temperature and decreasing the exfoliation time. 
Glycerol is a safe, polar solvent that has three hydroxyl functional groups and a hydrocarbon chain in its chemical structure. The boiling point of glycerol $\left(290^{\circ} \mathrm{C}\right)$ is higher than water $\left(100^{\circ} \mathrm{C}\right)$ which made it possible to increase the temperature of the process from $85^{\circ} \mathrm{C}$ to $250{ }^{\circ} \mathrm{C}$. This may accelerate the exfoliation process. Also, glycerol could cover the surface of the exfoliated particles and increase their affinity to interact with compositions within the fingermark residue.

As shown in Figure 1, after 1 hour stirring, the blue colour of the solution changed to brown. To investigate the reason for this change of colour, glycerol without any powder was stirred under the same condition for 3 days. The colour of the clear solution changed to yellow after $90 \mathrm{~min}$ and after 3 days the solution colour gradually turned to black (Figure 2). Therefore, change in the colour of the exfoliation mixture is due to the hot glycerol.
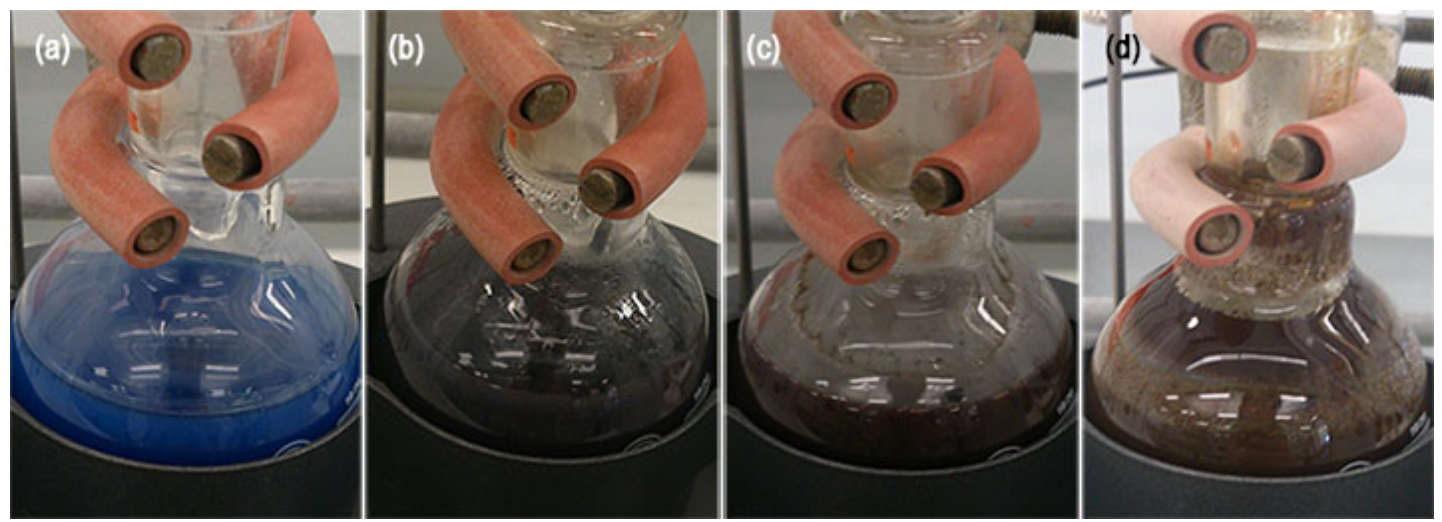

Figure 1 Photographs of the EB exfoliation process in glycerol photographed (a) before and (b-d) after exfoliation for (b) 1 hour, (c) 1 day and (d) 6 days.
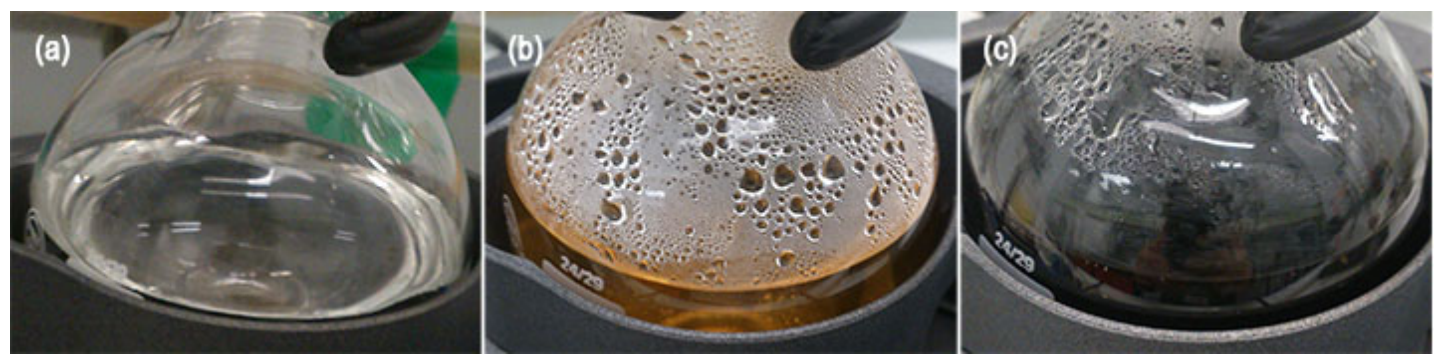

Figure 2 Photographs of glycerol heated under the same condition as the exfoliation process, (a) before and (b and c) after heating for (b) 90 min, (c) 3 days.

As shown in Figure 3, the dried exfoliated powder was made of two layers; a brown layer on top and a light blue layer underneath. The brown layer was cracked and peeled off after drying. The bottom layer was blue; therefore, the blue particles deposited sooner than the dark ones, which may indicate their larger size. Also, the reason could be that the brown particles were coated by a larger amount of glycerol compared to the blue ones and therefore remained suspended longer due to stabilisation. Brown particles mostly deposited after 1 day. The other reason could be 
oxidation of the top layer. Figure 4 shows that both layers are luminescent when excited at 590 $\mathrm{nm}$.

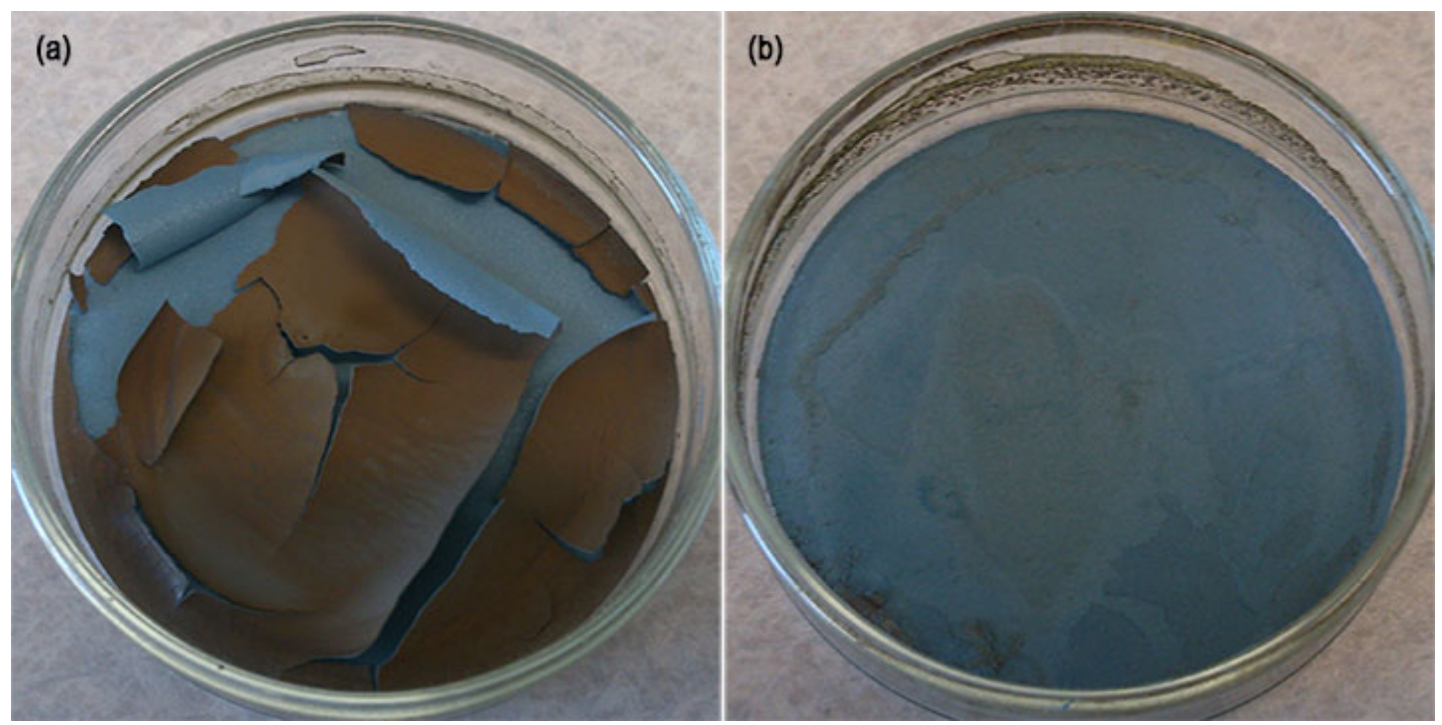

Figure 3 Dried exfoliated EB in glycerol (a) before and (b) after removing the brown layer.

Figure 5 shows optical photomicrographs indicating that 3-day exfoliated EB powder is a mixture of intact and reduced-size particles. Nine days exfoliation yielded less intact particles.

Zeta potential of the exfoliated particles was measured by dynamic light scattering (DLS) and demonstrated in Figure 6-a and b. These results reveal that the zeta potential of the particles did not change after exfoliation. Zeta potential of the exfoliated particles both in water stirred for 3 days (EEB-3D) and 11 days (EEB-11D), and glycerol stirred for 3 days and 9 days were compared in Figure 6-c. Exfoliation in water reduced the zeta potential (lower amount of negative charge). This reduction did not happen in exfoliation with glycerol. Preservation of high zeta potential is desired in terms of fingermark development since it could increase the electrostatic interaction between the exfoliated particles and composition within the fingermark secretion. 


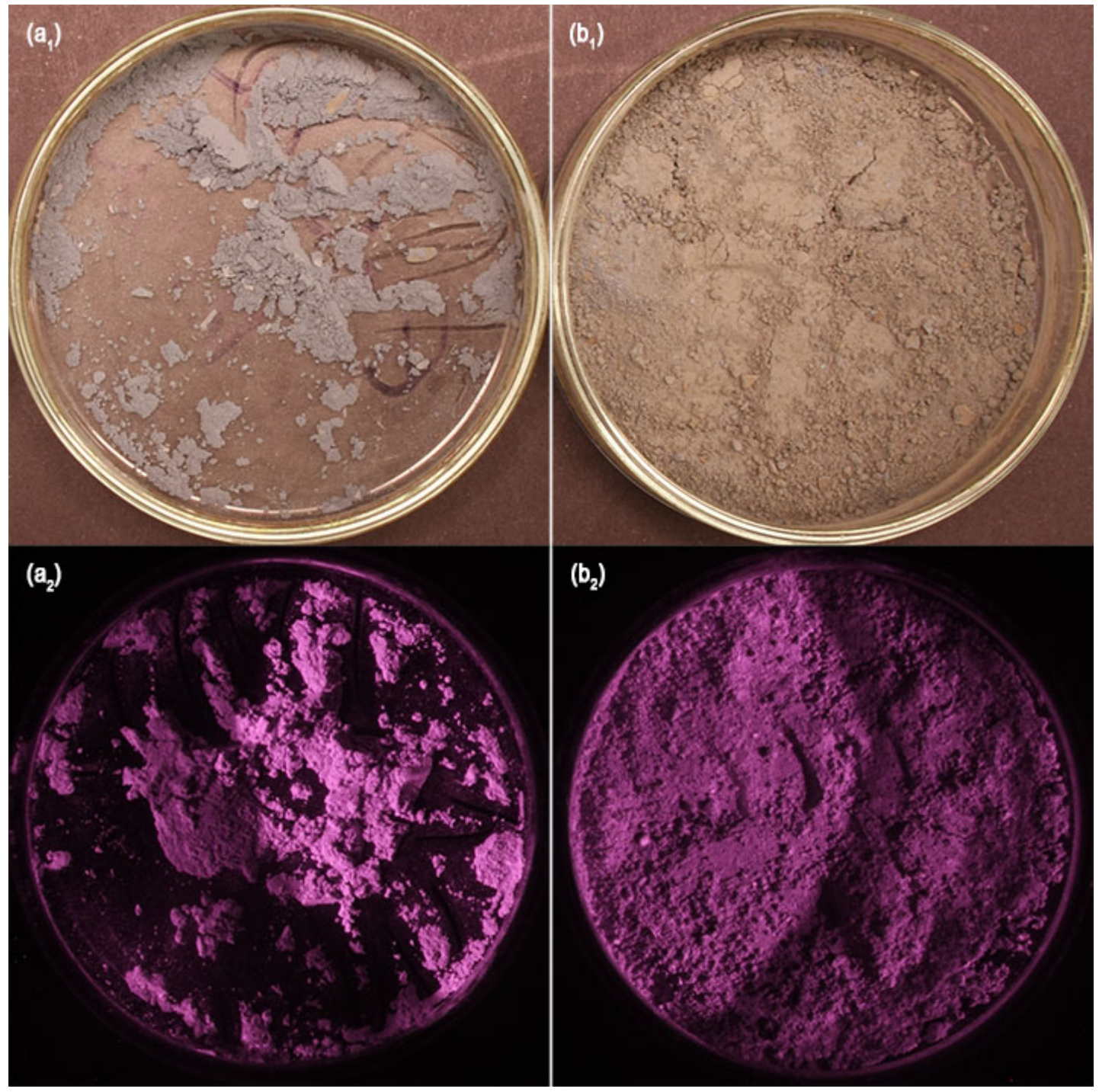

Figure 4 Photographs of (a) top layer and (b) bottom layers of the dried EEB-G-9D (see Figure 3); photographed by Canon camera under $\left(a_{1}\right.$ and $\left.b_{1}\right)$ ambient lighting (f-stop: $f / 9$, exposure time: $\left.1 / 2 \mathrm{~s}\right)$ and $\left(a_{2}\right.$ and $\left.b_{2}\right)$ illumination at $590 \mathrm{~nm}$ (IR long-pass filter, f-stop: $\mathrm{f} / 9$, exposure time: $2 \mathrm{~s}$ ). 


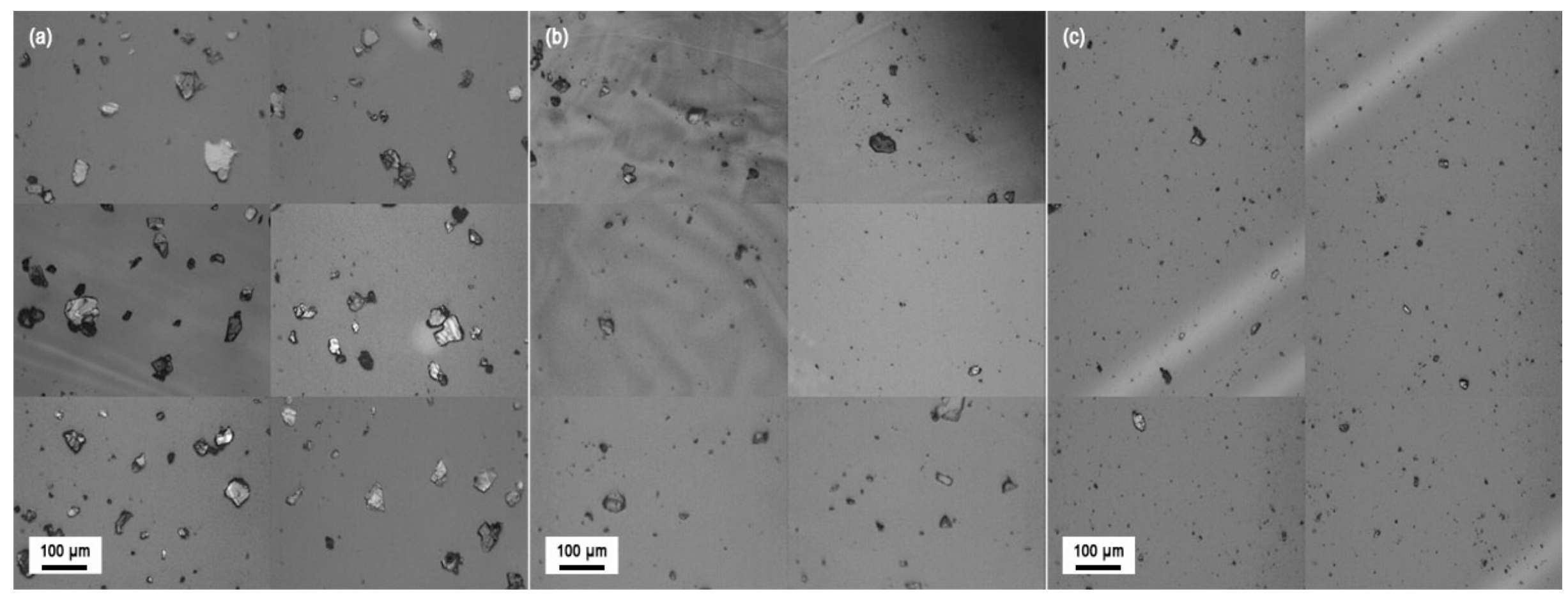

Figure 5 Images of (a) EB, (b) EEB-G-3D and (c) EEB-G-9D; captured by Nikon optical microscope. Each image is made of 6 images from different areas to show size distribution. 


\begin{tabular}{|c|c|c|}
\hline (a) & sample name & zeta potential $(\mathrm{mV})$ \\
\hline \multirow{5}{*}{ 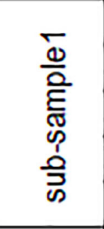 } & EEB-G-3D-11 & -32.2 \\
\hline & EEB-G-3D-1 2 & -30.9 \\
\hline & EEB-G-3D-1 3 & -29.8 \\
\hline & EEB-G-3D-1 4 & -29.3 \\
\hline & EEB-G-3D-1 5 & -30.1 \\
\hline \multirow{5}{*}{ 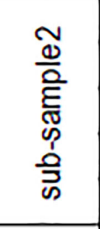 } & EEB-G-3D-2 1 & -33.8 \\
\hline & EEB-G-3D-2 2 & -33.9 \\
\hline & EEB-G-3D-2 3 & -31.2 \\
\hline & EEB-G-3D-2 4 & -30.4 \\
\hline & EEB-G-3D-2 5 & -25.4 \\
\hline \multirow{5}{*}{ 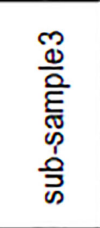 } & EEB-G-3D-3 1 & -34.9 \\
\hline & EEB-G-3D-3 2 & -34.1 \\
\hline & EEB-G-3D-3 3 & -34.6 \\
\hline & EEB-G-3D-3 4 & -34 \\
\hline & EEB-G-3D-3 5 & -33.7 \\
\hline mean & & -31.9 \\
\hline std dev & & 2.6 \\
\hline
\end{tabular}

\begin{tabular}{|c|c|c|}
\hline (b) & sample name & zeta potential $(\mathrm{mV})$ \\
\hline \multirow{5}{*}{ 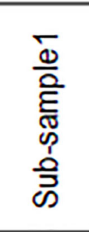 } & EEB-G-9D-1 1 & -33.3 \\
\hline & EEB-G-9D-1 2 & -33.1 \\
\hline & EEB-G-9D-1 3 & -31.6 \\
\hline & EEB-G-9D-1 4 & -31.4 \\
\hline & EEB-G-9D-1 5 & -30.5 \\
\hline \multirow{5}{*}{ 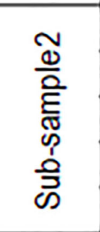 } & EEB-G-9D-2 1 & -31.6 \\
\hline & EEB-G-9D-2 2 & -30.6 \\
\hline & EEB-G-9D-2 3 & -30.9 \\
\hline & EEB-G-9D-2 4 & -29.6 \\
\hline & EEB-G-9D-2 5 & -31.8 \\
\hline \multirow{5}{*}{ 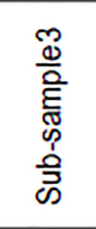 } & EEB-G-9D-3 1 & -28.8 \\
\hline & EEB-G-9D-3 2 & -28.5 \\
\hline & EEB-G-9D-3 3 & -29.6 \\
\hline & EEB-G-9D-3 4 & -30.9 \\
\hline & EEB-G-9D-3 5 & -29 \\
\hline mean & & -30.7 \\
\hline std dev & & 1.5 \\
\hline
\end{tabular}

\begin{tabular}{|c|c|}
\hline powder & zeta potential $(\mathrm{mV})$ \\
\hline EB & $-30.8 \pm 1.2$ \\
\hline EEB-3D & $-24.8 \pm 0.9$ \\
\hline EEB-11D & $-21.6 \pm 0.8$ \\
\hline EEB-G-3D & $-31.9 \pm 2.6$ \\
\hline EEB-G-9D & $-30.7 \pm 1.5$ \\
\hline
\end{tabular}

Figure 6 Zeta potential of the particles exfoliated in (a-c) glycerol and (c) water for the different durations; measured by DLS.

According to Figure 7, the exfoliated powders in glycerol were still luminescent in the nearinfrared (NIR) region when illuminated at $590 \mathrm{~nm}$ using a Polilight, however, expectedly, their luminosity decreased compared to the original powder. Photoluminescence (PL) spectra in Figure 8, show that the emission peak slightly blue-shifted from $916 \mathrm{~nm}$ to $906 \mathrm{~nm}$ after exfoliation. Also, the excitation peak slightly red-shifted and the peak at $590 \mathrm{~nm}$ disappeared after exfoliation. Figure 9 reveals that exfoliation of EB in glycerol did not noticeably affect fluorescent lifetime. 


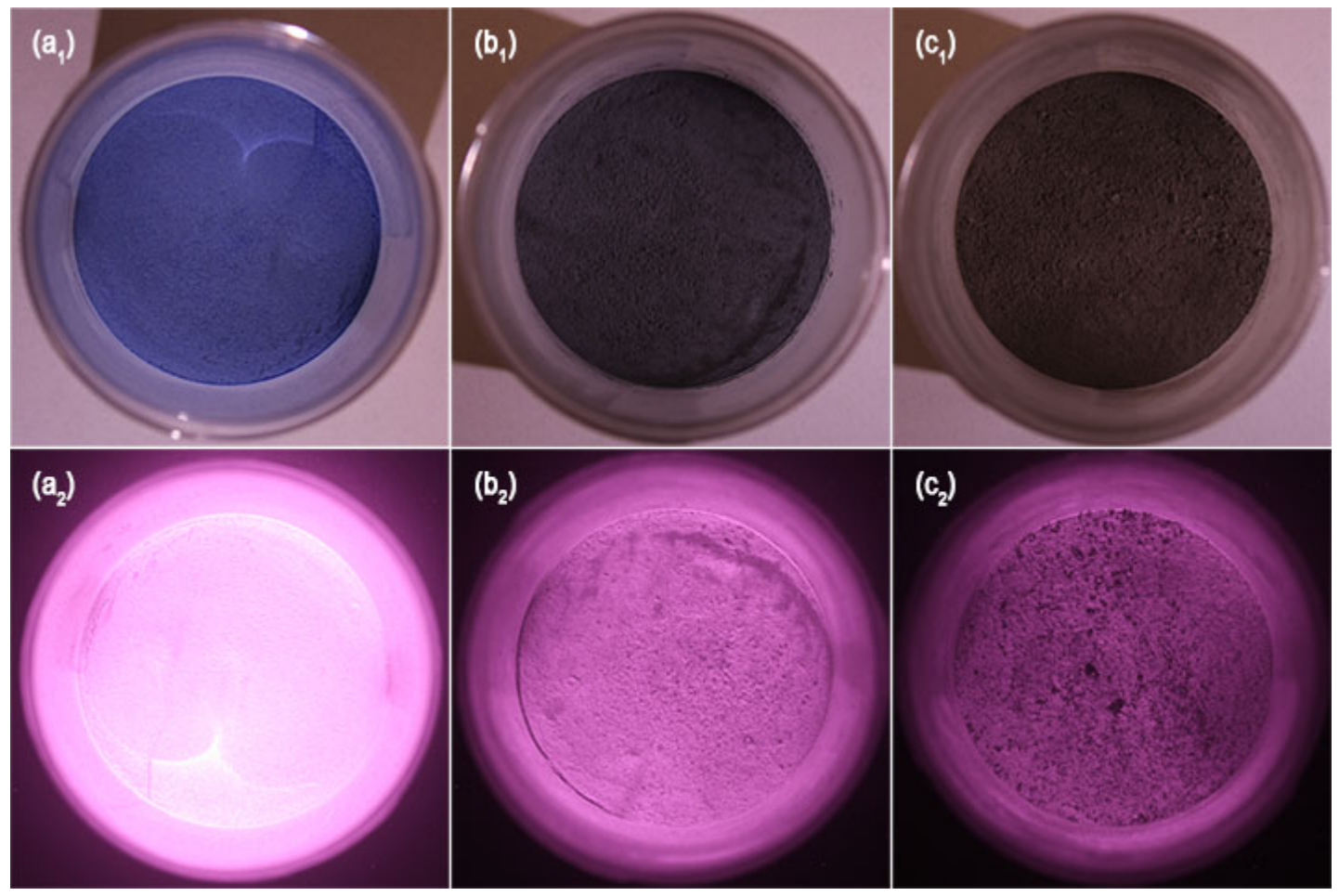

Figure 7 Photographs of (a) EB, (b) EEB-G-3D and (c) EEB-G-9D; photographed by Canon camera under $\left(a_{1}-c_{1}\right)$ ambient lighting (f-stop: $\mathrm{f} / 9$, exposure time: $1 / 2 \mathrm{~s}$ ) and $\left(\mathrm{a}_{2}-\mathrm{c}_{2}\right)$ illumination at $590 \mathrm{~nm}$ (IR long-pass filter, f-stop: $\mathrm{f} / 9$, exposure time: $2 \mathrm{~s})$.

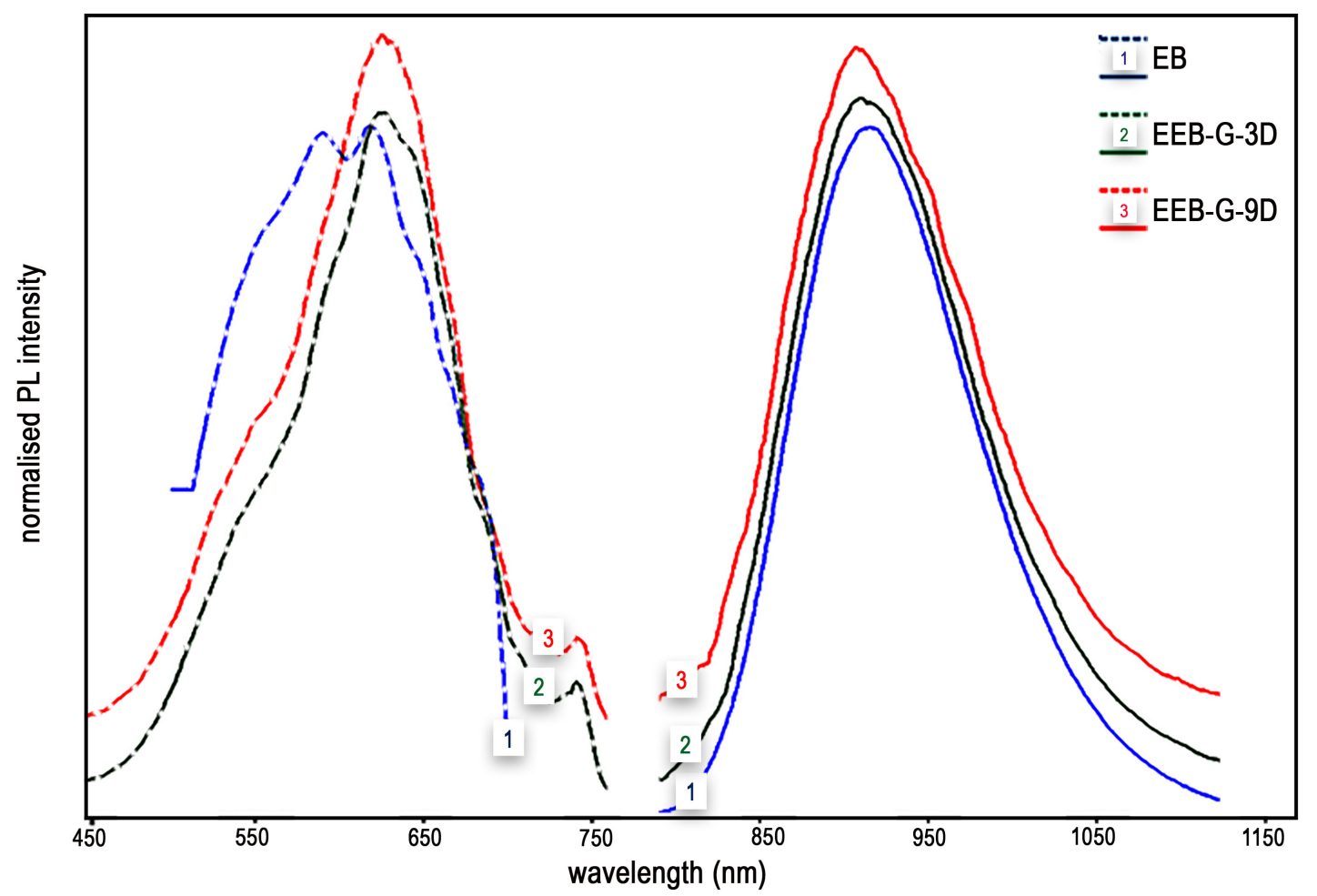

Figure 8 Luminescence emission (solid lines, $\lambda_{e x}=630 \mathrm{~nm}$ ) and excitation (dashed lines, $\lambda_{e m}=916 \mathrm{~nm}$ ) of EB pigments before and after exfoliation in glycerol for the different durations. Spectra were normalised to unity and have been offset for clarity. Spectra were smoothed using OPUS software based on the Savitzky-Golay algorithm. ${ }^{4}$ 


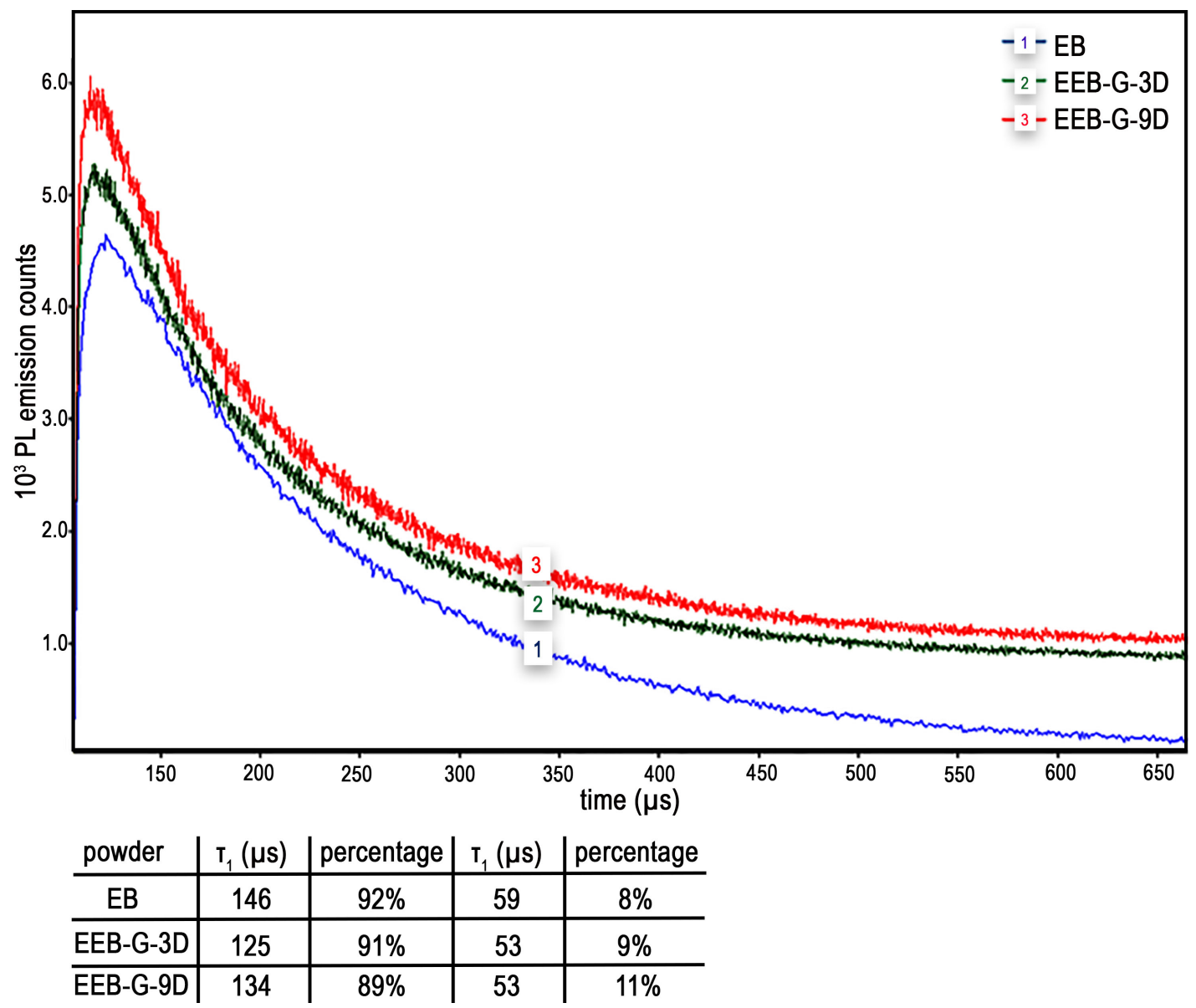

Figure 9 PL decay graphs of EB, EEB-G-3D and EEB-G-9D powders. Table shows measured fluorescent lifetimes based on the PL decay curves.

Particles size affects the intensity of the attenuated total reflectance (ATR) spectral peaks. ${ }^{5-8}$ Smaller particles could be compacted more tightly and fill the voids between them to increase this contact area and therefore increase absorption. ${ }^{5}$ Therefore, one technique to compare the particles size can be through their ATR spectrum. Figure 10 and Figure 11 illustrate the mid-infrared (MIR) and far-infrared (FIR) spectra collected by Fourier transform infrared spectroscopy (FTIR) from EB particles before exfoliation and after exfoliation in water and glycerol for the different durations.

As per previous reports, $\mathrm{EB}$ is made of several layers of tetrasilicates and $\mathrm{CuO}_{4}$ squares separated by $\mathrm{Cu}^{2+}$ ions. ${ }^{9-12}$ In MIR spectra (Figure 10), peaks between 420 and $1230 \mathrm{~cm}^{-1}$ have been reported in several studies for EB powder, ${ }^{13-16}$ which can be assigned to symmetrical and asymmetrical SiO-Si stretching. In FIR spectra (Figure 11), peaks at $520 \mathrm{~cm}^{-1}$ and $479 \mathrm{~cm}^{-1}$ can be assigned to CuO stretching. ${ }^{17,18}$ The peak at $479 \mathrm{~cm}^{-1}$ can also be assigned to O-Si-O bending vibrations. ${ }^{19,20}$ Peaks located within the wavenumbers of $360-300 \mathrm{~cm}^{-1}$ can be assigned to calcium oxide. ${ }^{21}$ 
In both spectral regions, 3-day exfoliated particles showed the greater spectral intensity of the peaks when particles were exfoliated in glycerol rather than water, indicating that smaller particles were achieved in a shorter time using glycerol. FIR spectra show almost the same peak intensity for 3-day and 9-day exfoliation in glycerol that are comparable to an 11-day exfoliation in water.

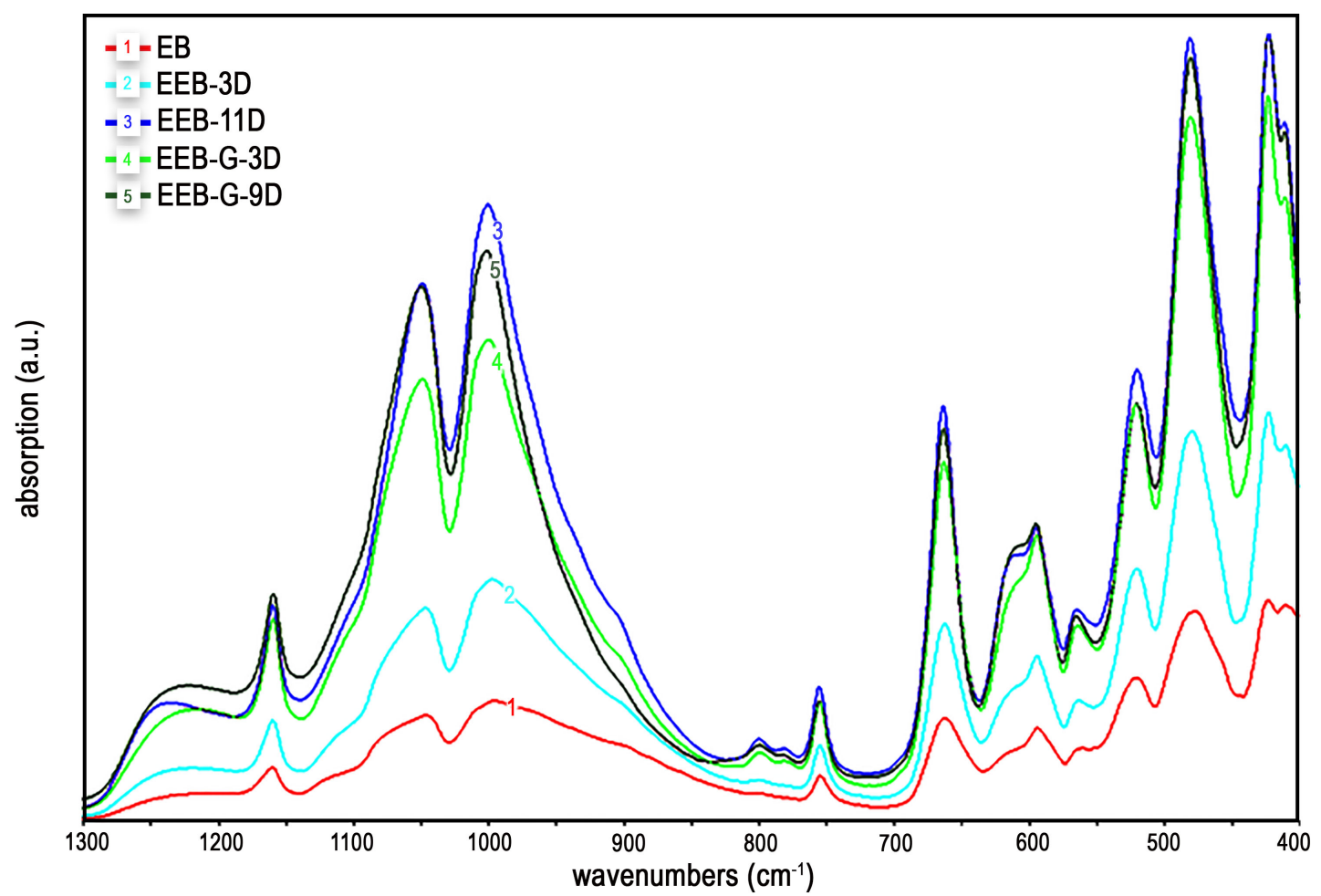

Figure 10 MIR spectra of EB as well as EEB in water and glycerol for the different durations. Data were collected by Nicolet FTIR. No intensity modification was applied. 


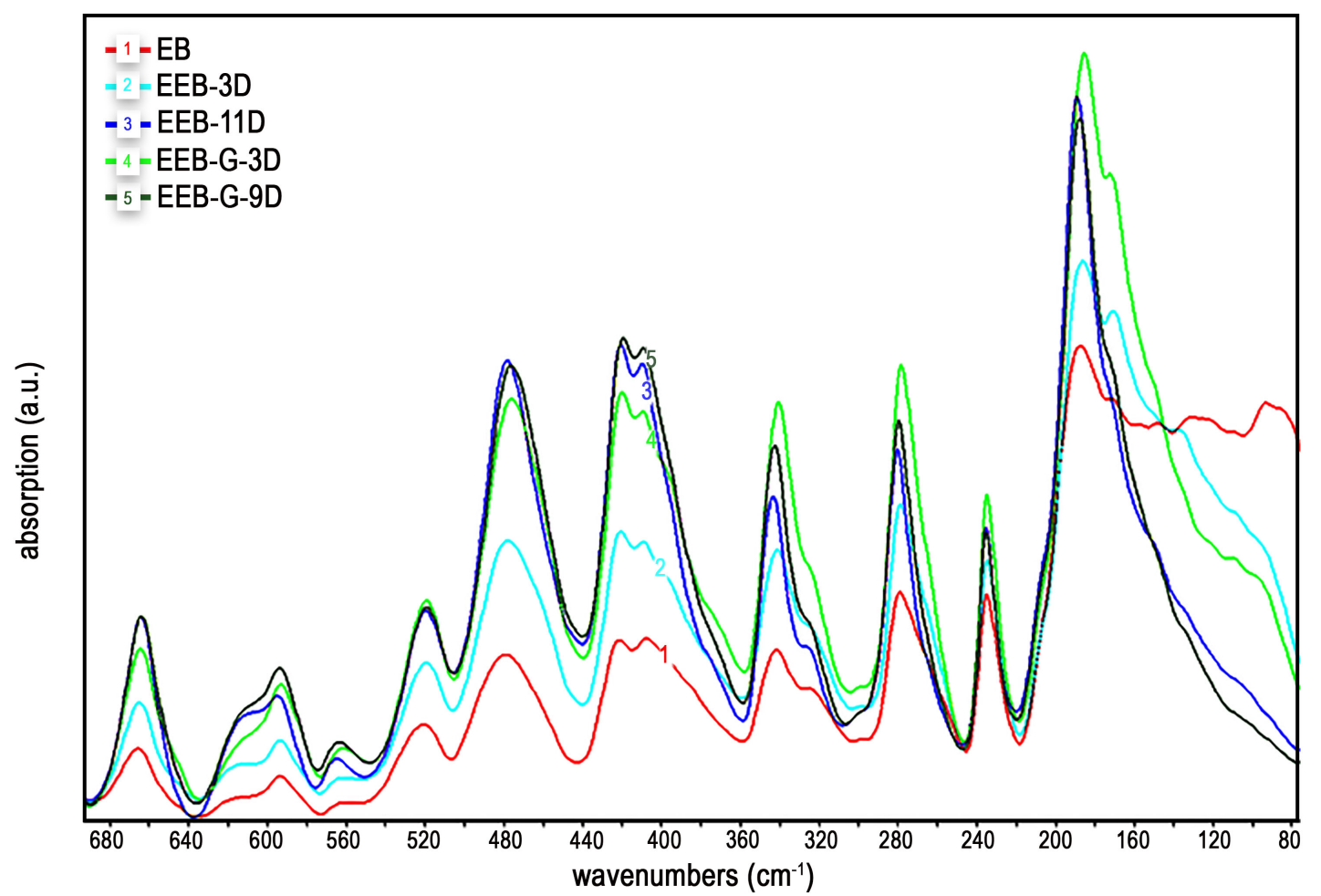

Figure 11 FIR spectra of EB as well as exfoliated EB in water and glycerol for the different durations. Data were collected by Bruker FTIR. No intensity modification was applied.

As discussed previously, the colour of the exfoliated particle was brown instead of light blue most likely because it contained a coating with dark glycerol even after washing. For further investigation, MIR spectra of glycerol were collected before (clear solution) and after (dark solution) heating (Figure 2). Figure 12 illustrates the spectra. The only difference is the appearance of a peak at $1732 \mathrm{~cm}^{-1}$ after heating. This peak could be assigned to $\mathrm{C}=\mathrm{O}$ stretching of carboxyl group indicating oxidation of glycerol.

MIR spectra of unexfoliated EB and exfoliated EB in glycerol for 3 and 9 days were compared with heated glycerol in Figure 13 to investigate whether the glycerol coated the particles or not. As illustrated in Figure 13-b-d, several new peaks within the ranges of $3650-2850 \mathrm{~cm}^{-1}$ and 1800 $1350 \mathrm{~cm}^{-1}$ appeared after exfoliation in glycerol. These peaks could be assigned to $\mathrm{OH}$ stretching, symmetric and asymmetric $\mathrm{CH}_{2}$ stretching, $\mathrm{CH}_{2}$ bending and wagging as well as $\mathrm{OH}$ bending which existed in the spectrum of glycerol as well. ${ }^{22}$ These peaks shifted compared to the peaks in the heated glycerol spectrum, which indicates interaction between oxidised glycerol and surface of the particles through hydroxyl and carboxyl groups. Also, peaks at $1047 \mathrm{~cm}^{-1}$ and $996 \mathrm{~cm}^{-1}$ in EB powder shifted after exfoliation, these peaks could be assigned to asymmetric Si-O-Si stretching. ${ }^{12}$ 


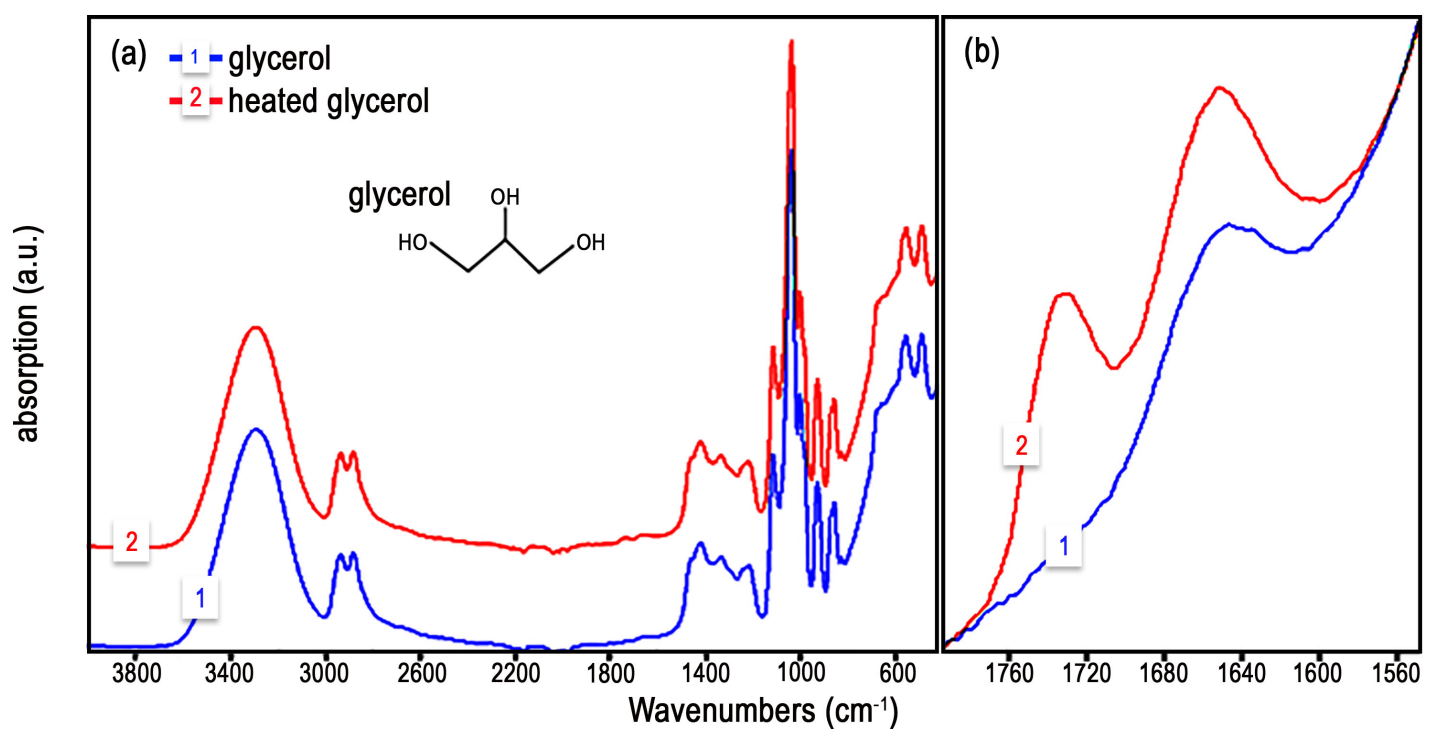

Figure 12 (a) MIR spectra of glycerol before and after heating. (b) Spectra in narrower ranges of wavenumbers; collected by Nicolet FTIR.

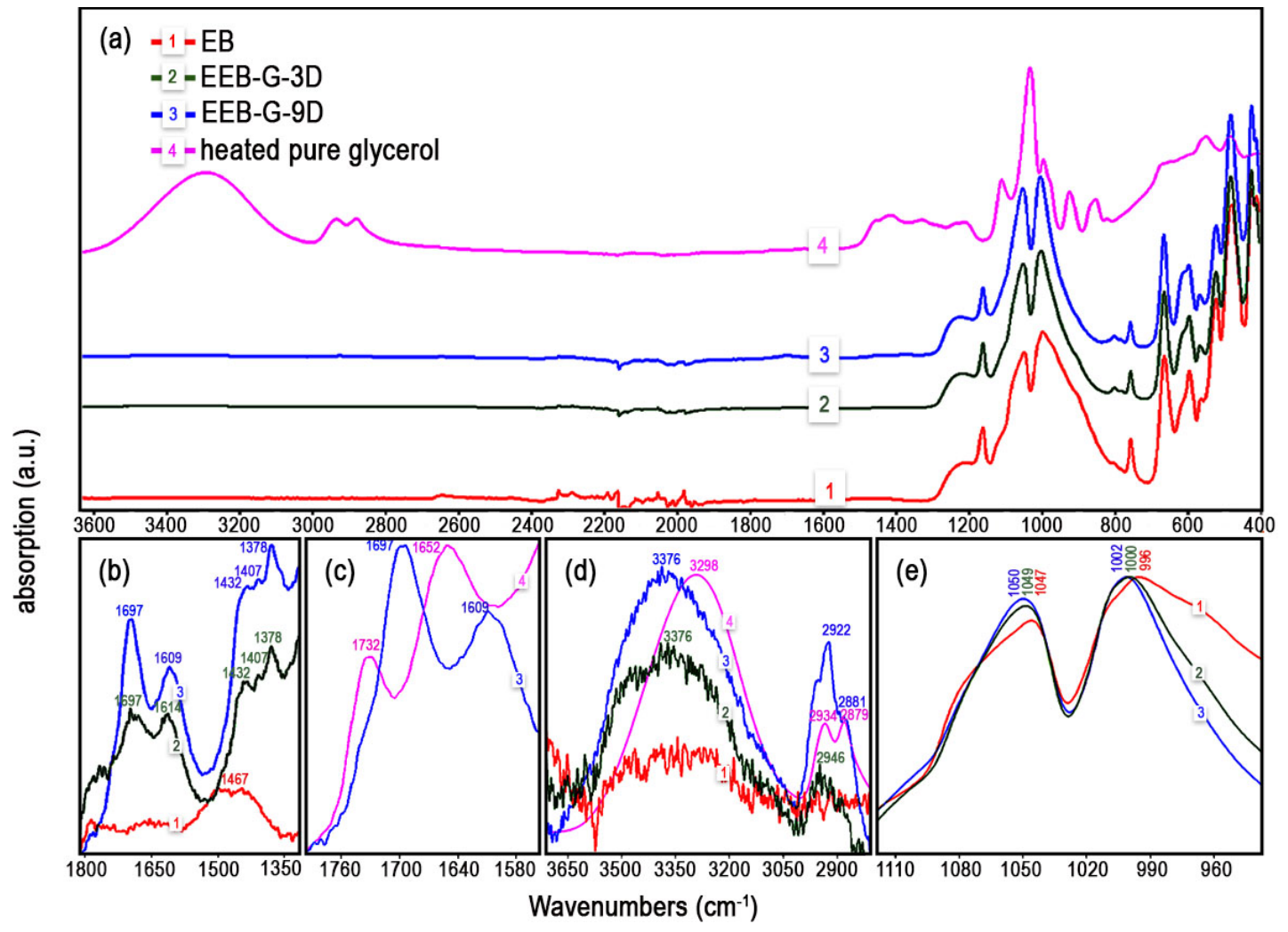

Figure 13 (a) MIR spectra of EB before and after exfoliation in glycerol and heated glycerol. (b-e) Spectra in narrower ranges of wavenumbers; collected by Nicolet FTIR.

\subsubsection{Fingermarks visualisation}

First, it should be mentioned that a particular advantage of using the glycerol exfoliation method is the generation of visually darker fingermark dusting powder. The dark colour of exfoliated particles in glycerol makes it possible for the naked eye to visualise the treated latent fingermarks 
on light-coloured substrates, while the light blue water-exfoliated particles could be used for the marks on dark-coloured substrates.

An experiment on depletion series of natural latent fingermarks from 2 donors deposited on glass slides was conducted. Split fingermarks were treated by EB exfoliated in water and glycerol (longterm stirred; 9 days in glycerol and 11 days in water). Results in Figure 14 demonstrate that highquality luminescent fingermarks even in $4^{\text {th }}$ depletions were achieved using both powders.

In another experiment, long-term and short-term stirred EB in glycerol and water were compared. Natural fingermarks from 6 donors were deposited on glass slides and treated with 3-day and 9day exfoliated EB in glycerol as well as 3-day and 11-day exfoliated EB in water. It should be noted that the fingermark secretion from the same donor was used for all four dusting powders. According to Figure, fingermarks developed with short-term stirred EB in glycerol had higher qualities since they have a combination of desired characteristics including size, luminosity and surface modification. Unfortunately, they had a high affinity to stick to the glass substrate and developed the background as well. 
depletion 1

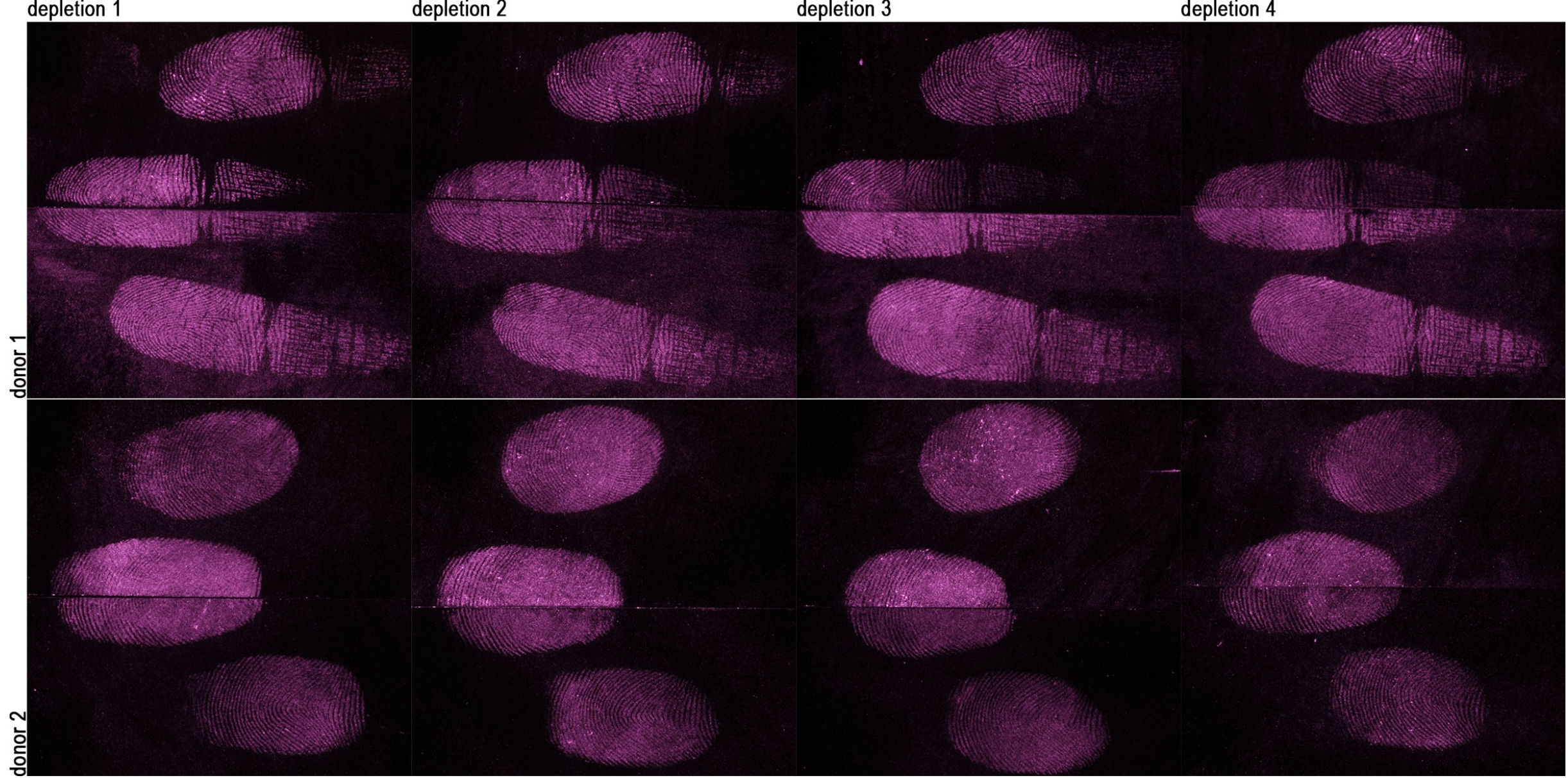

Figure 14 Comparison between EB exfoliated in glycerol and water: 4 depletions of 3-day old natural split fingermarks from 2 female donors were deposited on glass slides, treated with EEB-G-9D (lower half) and EEB-11D (upper half) and photographed with Canon camera (excitation: $590 \mathrm{~nm}$, IR long-pass filter, f-stop: f/9, exposure times: donor 1: $15 \mathrm{~s}$ and donor $2: 8 \mathrm{~s})$. 


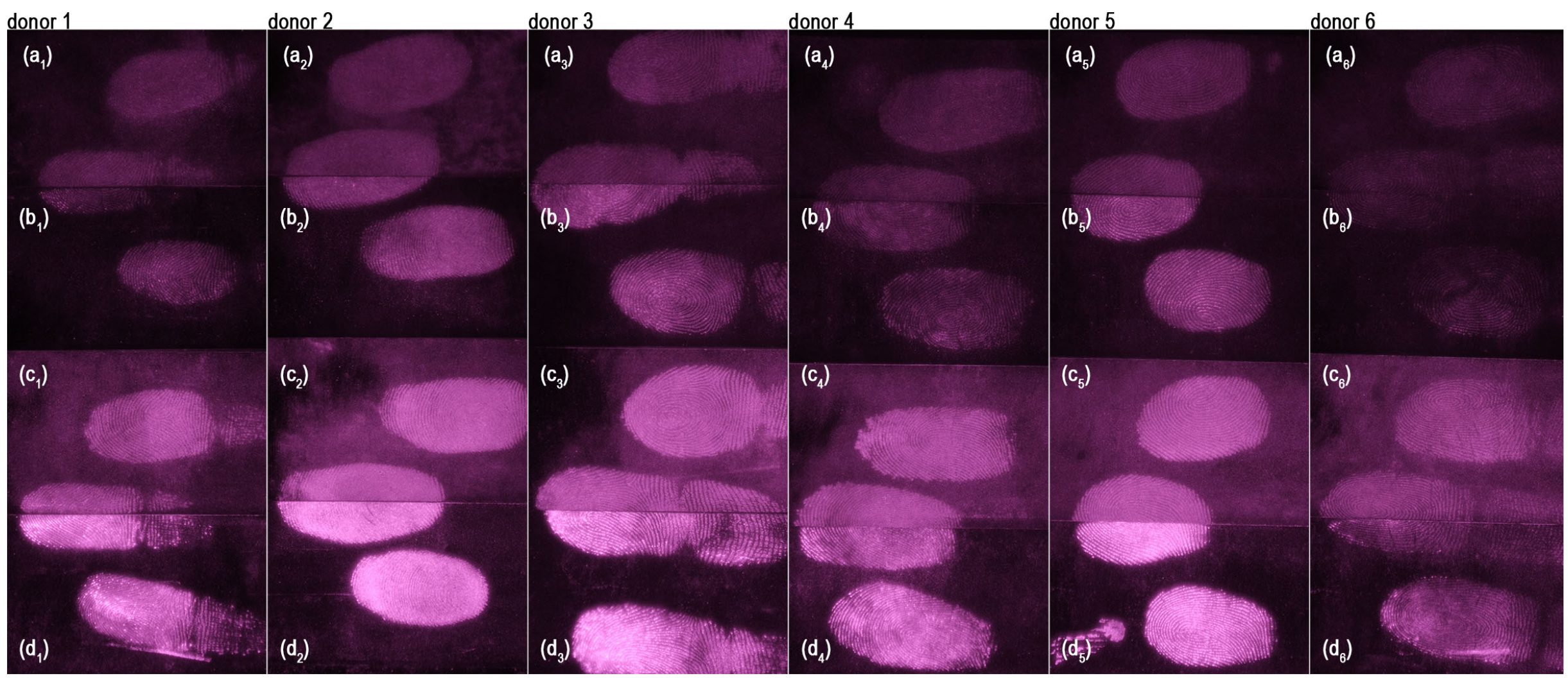

Figure15 Comparison between EB exfoliated in glycerol and water for different duration: 2-day old natural split fingermarks from 6 donors (3 female and 3 male) deposited on glass slides and treated with (a) EEB-G-9D, (b) EEB-11D, (c) EEB-G-3D and (d) EEB-3D; photographed with Canon camera (excitation: 590 nm, IR long-pass filter, f-stop: f/9, exposure time: $5 \mathrm{~s})$. 


\subsection{Exfoliation of Egyptian blue powder in methanol and ethanol}

To simplify further the exfoliation technique, heating was eliminated while methanol and ethanol were used as solvents in combination with a cationic surfactant.

\subsection{Experiment}

A mixture of $0.5 \mathrm{~g}$ of EB powder (Kremer 10600) and $0.15 \mathrm{~g}$ of cetyltrimethylammonium bromide (CTAB) powder was vigorously stirred in $40 \mathrm{~mL}$ of methanol or ethanol in a capped bottle at room temperature for 13 days. The mixture was left overnight at room temperature to let the powder settle and the supernatant became clear (centrifuge could be used as well). The supernatant was decanted and the powder left to dry overnight at room temperature and then 3 days in an oven $\left(30^{\circ} \mathrm{C}\right) . \mathrm{CTAB}$ coated exfoliated $\mathrm{EB}$ in methanol $(\mathrm{EEB}+\mathrm{C}-\mathrm{M})$ and ethanol $(\mathrm{EEB}+\mathrm{C}-\mathrm{E})$ were used for further experiments.

\subsection{Results and discussion}

To simplifying the preparation of exfoliated EB, heating the exfoliation solvent was eliminated. As mentioned previously, other than temperature, the polarity of the solvent and ionic surfactant are important factors in exfoliation of layered structures. Based on previous experiments and other reports, CTAB is a suitable ionic surfactant for exfoliation. ${ }^{3}$ It is also desirable in terms of fingermark development because of its ability to interact favourably with fingermark secretions. Methanol and ethanol are highly polar solvents and due to the hydrocarbon chain in their structure, both dissolve CTAB easily at room temperature. Figure 16 shows that at room temperature, CTAB dissolved in methanol immediately, however, a mixture of CTAB in water was not clear even after one-day stirring. Therefore, methanol and ethanol containing CTAB were used to exfoliate EB particles at room temperature.

Figure 17 shows the exfoliation mixture during the experiment. The bright blue colour of the EB particles lightened over time as an indication of successful exfoliation. However, the colour of the mixture in methanol got lighter sooner than ethanol (after 2 days). Also, the colour of the final product prepared in methanol was lighter than the one prepared in ethanol. Images acquired by optical microscopy in Figure 18 reveal that the size of particles decreased noticeably by exfoliation in methanol and ethanol. 


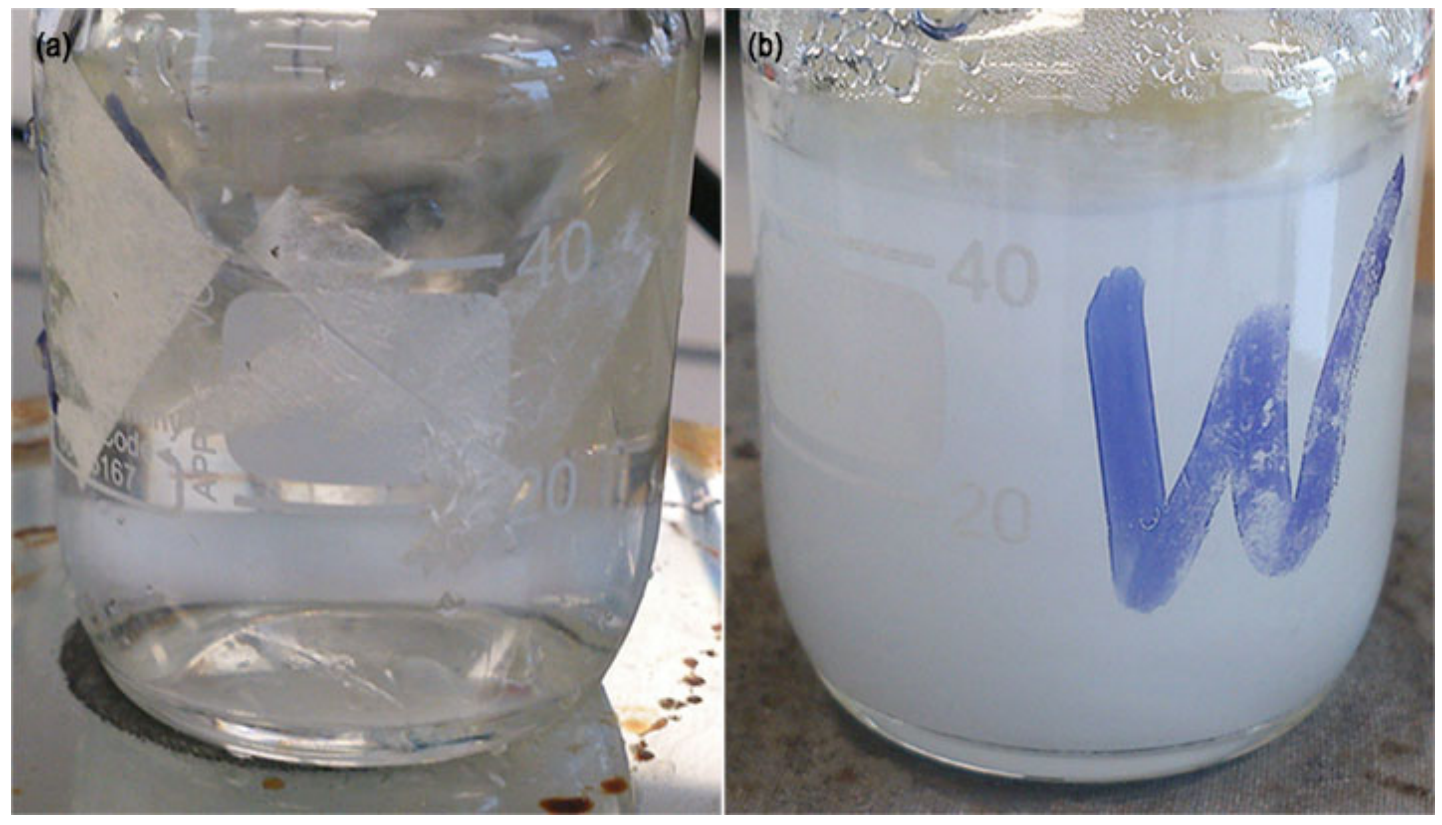

Figure 16 Photographs of CTAB stirred in (a) methanol and (b) water at room temperature.

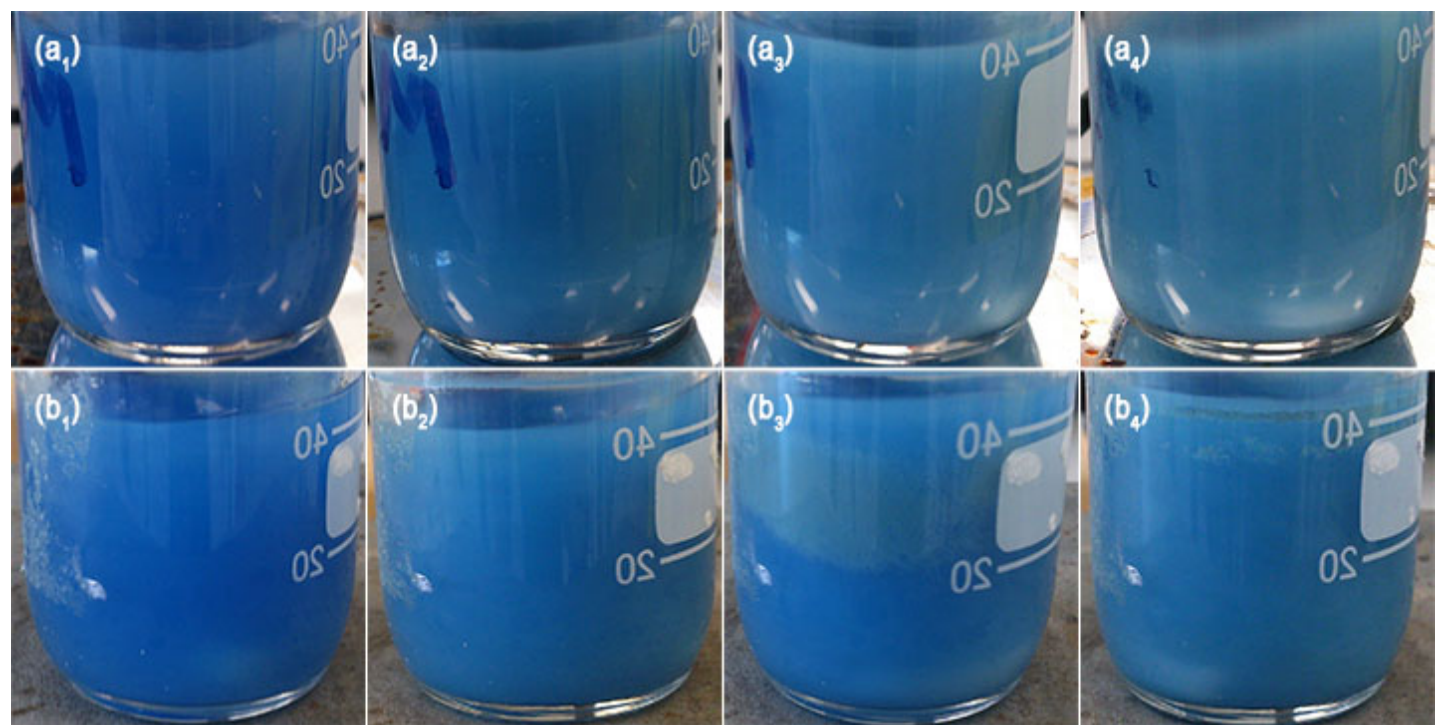

Figure 17 Photographs of the process of EB exfoliation in (a) methanol and (b) ethanol photographed $\left(a_{1}\right.$ and $\left.b_{1}\right)$ before and after exfoliation for $\left(a_{2}\right.$ and $\left.b_{2}\right) 1$ day, ( $a_{3}$ and $\left.b_{3}\right) 2$ days and $\left(a_{4}\right.$ and $\left.b_{4}\right) 13$ days. 


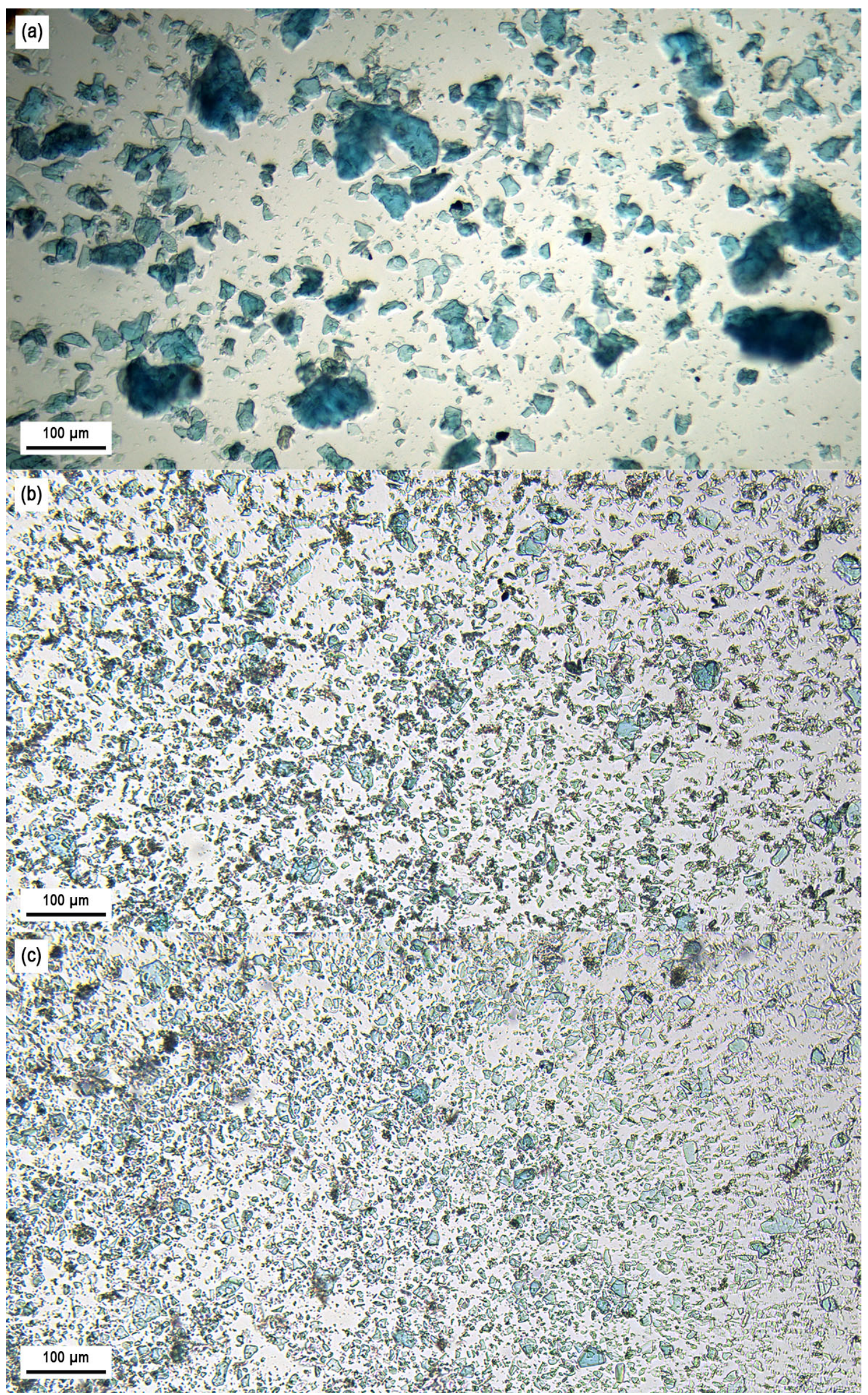

Figure 18 Images of (a) EB particles and exfoliated EB stirred in (b) ethanol and (c) methanol; captured by Leica optical microscope. 
FIR and MIR spectra of EB before and after exfoliation in methanol and ethanol are illustrated in Figure 19 and Figure 20. The peak intensity increased after exfoliation in both methanol and ethanol indicating a decrease in the size of the particles. However, the absorbance of particles exfoliated in methanol was larger than of the particles exfoliated in ethanol, which could indicate that methanol exfoliated particles more effectively than ethanol. This result confirms the aforementioned lighter colour of the particles exfoliated in methanol rather than ethanol (Figure 17). A reason for this may be the higher polarity of methanol due to a shorter hydrocarbon chain compared to ethanol.

Figure 20-b shows that symmetric and asymmetric stretching peaks of C-H in the exfoliated EB from the adsorbed CTAB appeared in the MIR spectra after exfoliation. These peaks slightly shifted compared to the peaks in the pure CTAB spectrum, which indicates interaction between CTAB and the particles. As per Figure 20-c, another peak appeared after exfoliation around 1200 $\mathrm{cm}^{-1}$ near one of the major peaks of CTAB. Also, the peak at $1160 \mathrm{~cm}^{-1}$ shifted after exfoliation. As the last difference, the peaks at $1462 \mathrm{~cm}^{-1}$ and $1463 \mathrm{~cm}^{-1}$ appeared in ethanol and methanol spectra, respectively instead of two peaks at $1498 \mathrm{~cm}^{-1}$ and $1444 \mathrm{~cm}^{-1}$ in EB spectrum (Figure 20d).

As a proof of concept, a 6-day old natural fingermark deposited on a ceramic tile was successfully visualised with coated EB particles exfoliated in methanol (Figure 21).

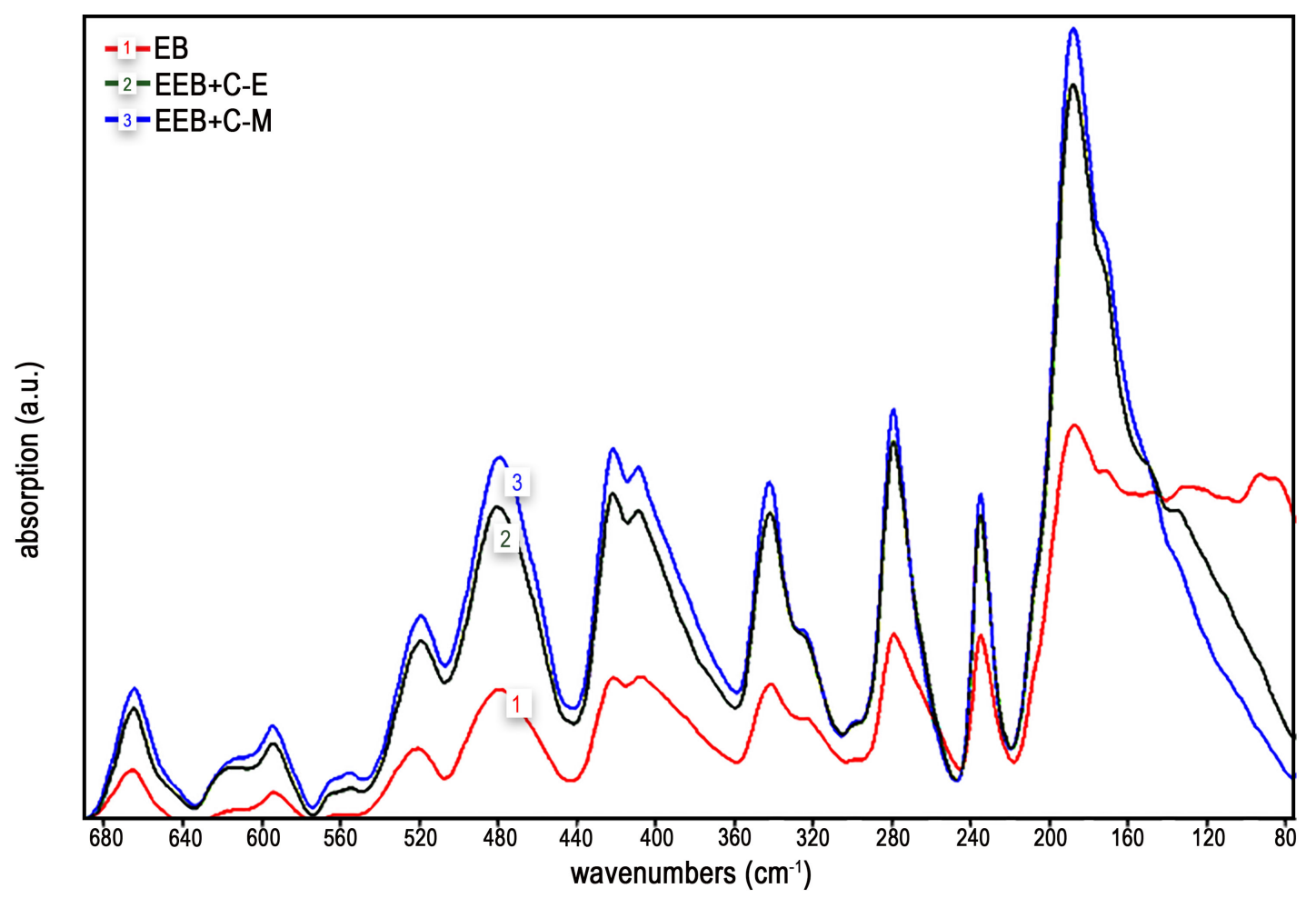


Figure 19 FIR spectra of EB as well as EB exfoliated in methanol and ethanol; collected by Bruker FTIR. No intensity modification was applied.

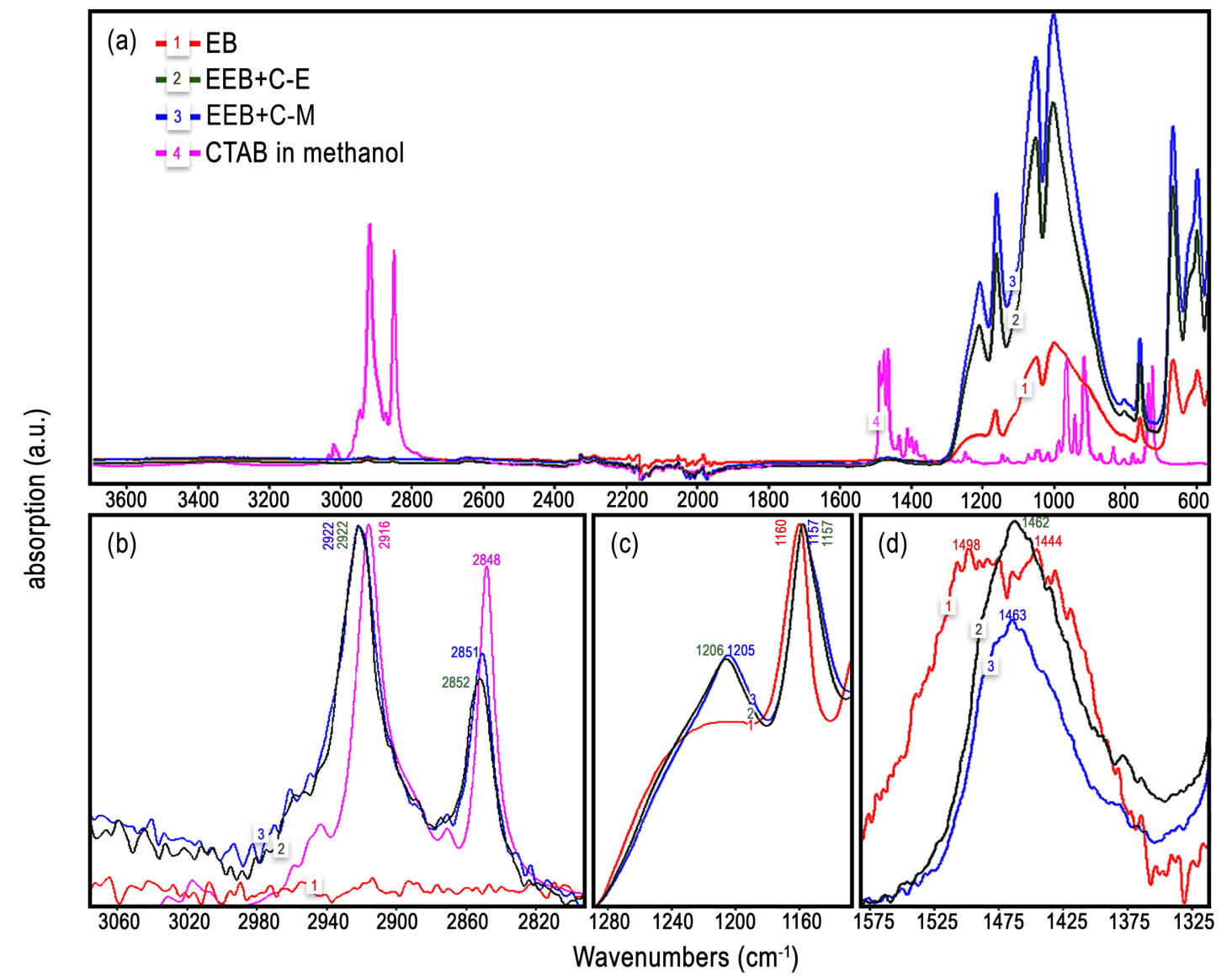

Figure 20 MIR spectra of EB before and after exfoliation in methanol and ethanol as well as CTAB in methanol. (a) No intensity modification was applied. (b-d) Spectra in narrower ranges of wavenumbers; collected by Nicolet FTIR. 


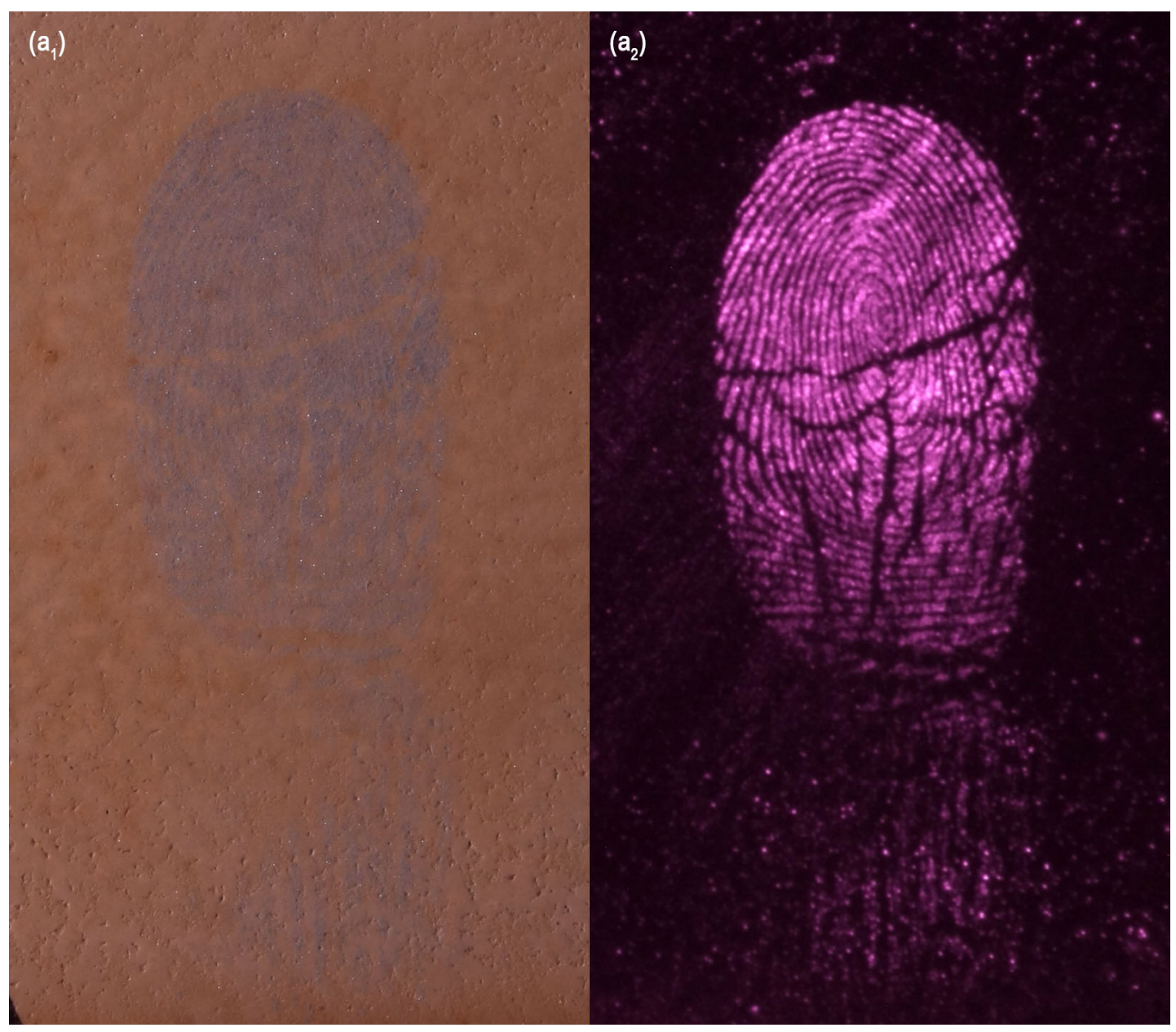

Figure21 6-day old natural fingermarks from a female donor deposited on ceramic tile, treated with EEB+C-M and photographed with Canon camera under $\left(\mathrm{a}_{1}\right)$ ambient lighting (f-stop: $\mathrm{f} / 8$, exposure time: $\left.1 / 400 \mathrm{~s}\right)$ and $\left(\mathrm{a}_{2}\right)$ illumination at $590 \mathrm{~nm}$ (IR long-pass filter, f-stop: $\mathrm{f} / 8$, exposure time: $3.2 \mathrm{~s}$ ).

\subsection{Exfoliation of Egyptian blue powder in polyethylene glycol}

The same procedure as exfoliation in glycerol was used to exfoliate Egyptian blue in polyethylene glycol 400 (PEG) at $200{ }^{\circ} \mathrm{C}$ for 11 days (EEB-P). The long hydrocarbon chain in the structure of PEG affects its polarity and renders it a less polar solvent compared to water and glycerol. Images of the particles acquired by the optical microscope (Figure 23 and Figure 18-a) and the intensity of peaks in MIR spectra (Figure 22) reveal that negligible change in the size of the particles occurs which highlights the role of the polarity of the solvent in the exfoliation process. 


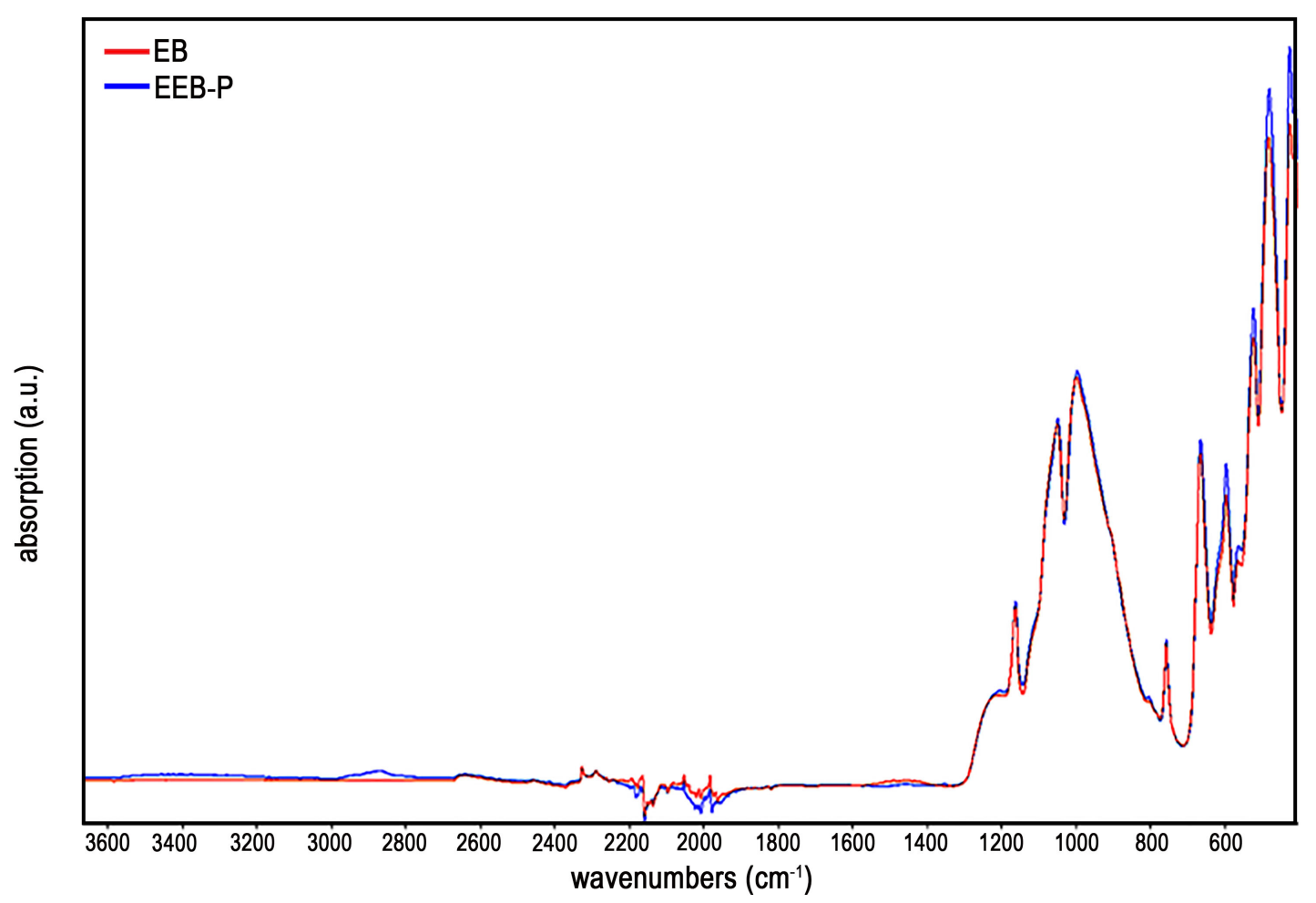

Figure 22 MIR spectra of EB before and after exfoliation in PEG; collected by Nicolet FTIR.

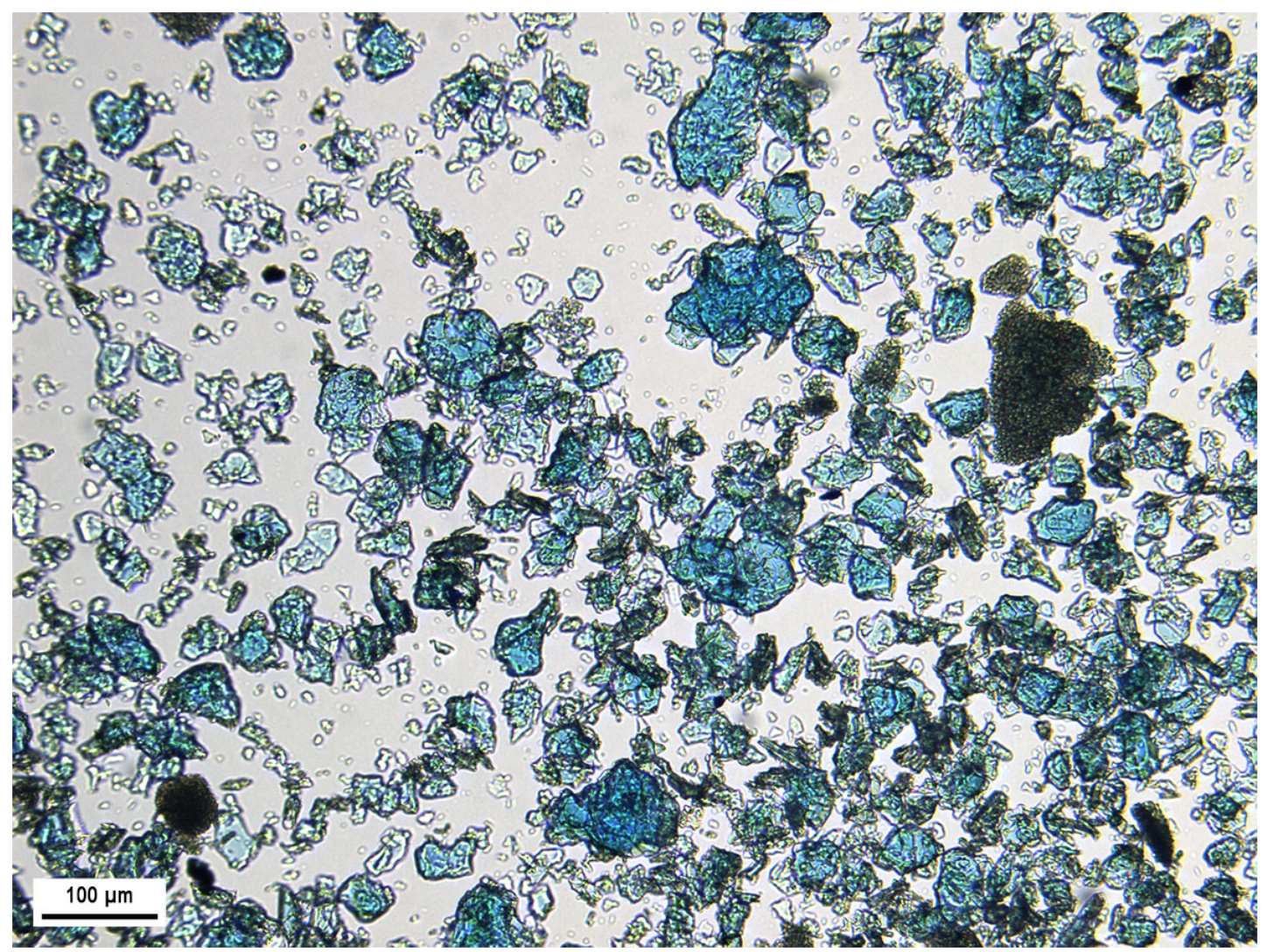

Figure 23 Image of EEB-P particles captured by the Leica optical microscope. 


\subsection{Exfoliated Egyptian blue powder coated with Tween 20}

Tween 20 (Polysorbate 20, $\mathrm{C}_{58} \mathrm{H}_{114} \mathrm{O}_{26}$, inset in Figure 24) as a non-ionic surfactant was used in previous reports as a surfactant to coat nanoparticles applied for the development of latent fingermarks. ${ }^{23-26}$ It contains long hydrocarbon chain as well as hydroxyl and carboxyl functional groups, which can target components within the secretion.

The same procedure as for exfoliation of EB in water was used. Four millilitres of Tween 20 was added to the solution instead of CTAB. Figure 24 shows the MIR spectra of EB, exfoliated Egyptian blue in water with (EEB+T20) and without Tween 20 as well as pure Tween 20. Figure 24-b-c show the appearance of new peaks and shifting in the peaks after addition of Tween 20 , which indicates the interaction of Tween 20 with the surface of the particles.

Figure 25 compares fresh charged fingermarks developed with EB powder exfoliated in the presence of Tween 20 and without Tween 20. A larger amount of coated particles adhered to the fingermarks compared to the uncoated particles. In the next step, fingermarks were gently brushed with a feather brush to remove excess powder from the fingermark and the substrate. The illuminated fingermark treated with uncoated exfoliated EB in Figure 25-a 3 illustrates less luminosity after brushing because the particles were easily removed from the secretion. However,

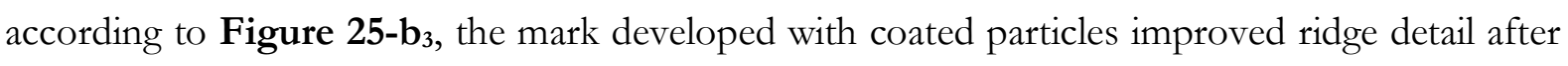
brushing away surface excess, which indicates stronger adherence of the lipophilic coated particles to the fingermark residues. 


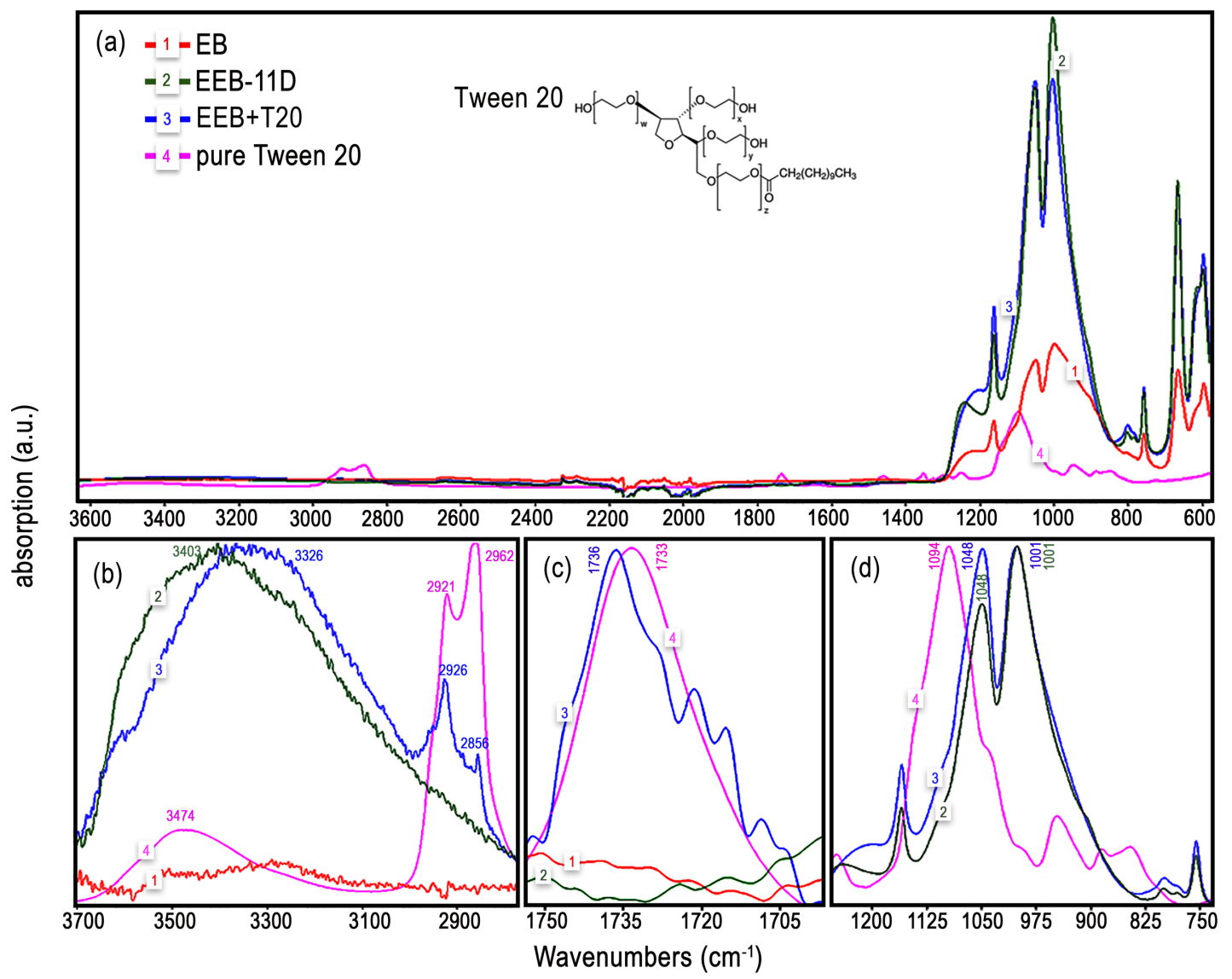

Figure 24 MIR spectra of EB before and after exfoliation in water and water+Tween 20 as well as pure Tween 20.

(a) No intensity modification was applied except for pure Tween 20 (b-d) Spectra in narrower ranges of wavenumbers; collected by Nicolet FTIR. Inset in (a) shows Tween 20 chemical structure adapted from https://www.sigmaaldrich.com. 


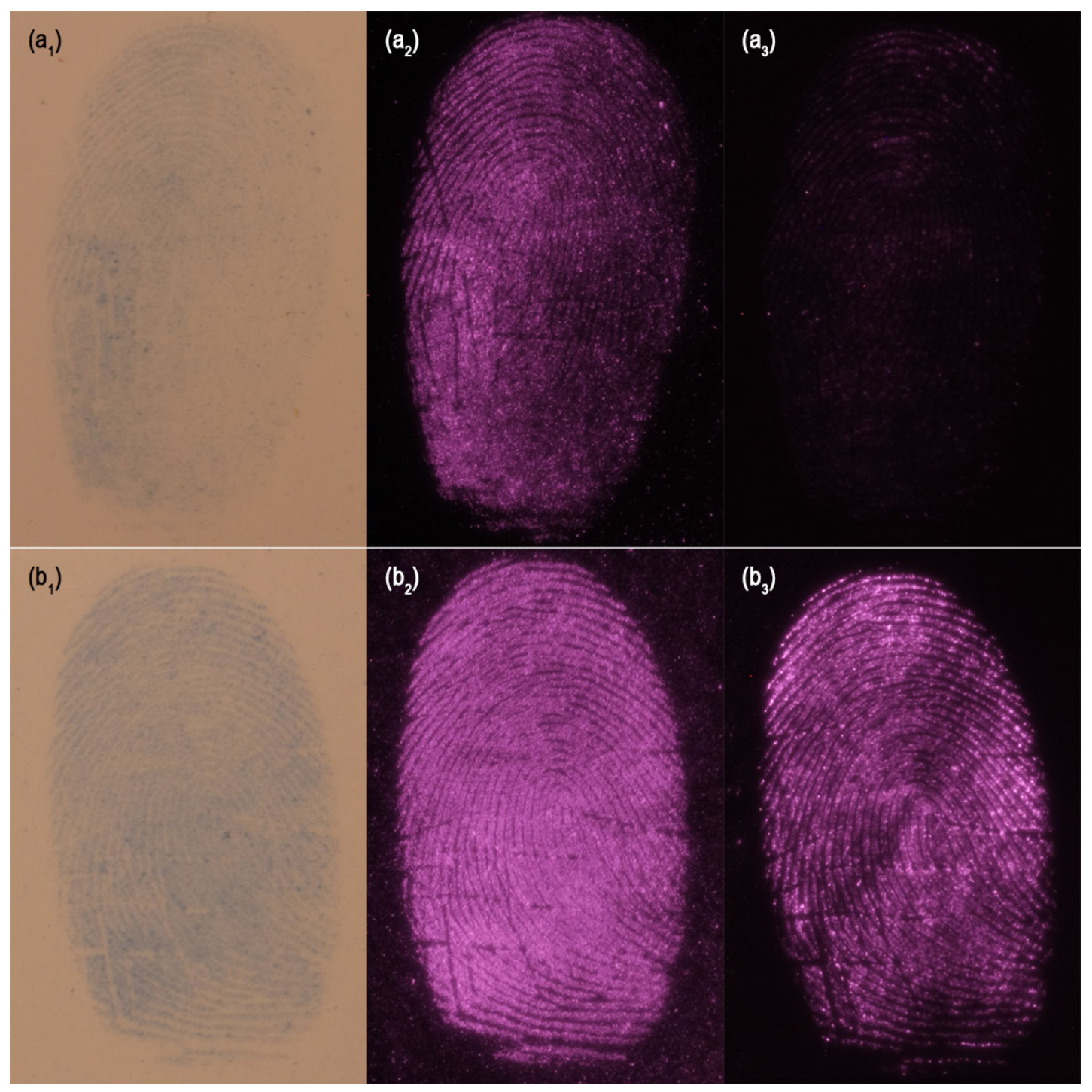

Figure 25 Comparison between Tween 20-coated and uncoated exfoliated EB: fresh charged fingermarks from a female donor deposited on white tiles, treated with (a) EEB-11D and (b) EEB+T20 and photographed with Canon camera under $\left(a_{1}\right.$ and $\left.b_{1}\right)$ ambient lighting (f-stop: $f / 2.4$, exposure time: $\left.1 / 100 \mathrm{~s}\right)$ and $\left(a_{2}, a_{3}, b_{2}\right.$ and $\left.b_{3}\right)$ illumination at $590 \mathrm{~nm}$ (IR long-pass filter, f-stop: $\mathrm{f} / 11$, exposure time: $5 \mathrm{~s}$ ). ( $\mathrm{a}_{3}$ and $\mathrm{b}_{3}$ ) Gently brushed fingermarks after treatment.

\section{CRediT authorship contribution statement}

Sorour Shahbazi: Conceptualization, Methodology, Investigation, Visualisation, Writing-original draft, Writing-review \& editing. John V. Goodpaster: Conceptualization, Writing-review \& editing. Gregory D. Smith: Conceptualization, Writing-review \& editing. Thomas Becker: Writing-review \& editing, Supervision. Simon W. Lewis: Conceptualization, Supervision, Project administration. 


\section{Acknowledgements}

The authors would like to acknowledge Dr. Mark Hackett (Curtin University) and Dr. Amanda Frick (Curtin University, now at Northumbria University) for their support during this research. We also thank Dr Kari Pitts (ChemCentre) for her help with optical microscopy. The authors thank all of the fingermark donors for their cooperation. J. V. Goodpaster thanks the IUPUI School of Science and IUPUI Sabbatical Leave Committee for their support of his time at Curtin University. S. Shahbazi was supported by an Australian Postgraduate Award. The authors declare no competing financial interest. This research has been conducted in accordance with the Curtin University Human Research Ethics Committee (Approval Number HRE2016-0252).

\section{References}

1. Shahbazi, S., et al., Preparation, characterization, and application of a lipophilic coated exfoliated Egyptian blue for nearinfrared luminescent latent fingermark detection. Forensic Chemistry, 2020. 18: p. 100208.

2. Nicolosi, V., et al., Liquid exfoliation of layered materials. Science, 2013. 340(6139): p. 1226419.

3. Backes, C., et al., Guidelines for exfoliation, characterization and processing of layered materials produced by liquid exfoliation. Chemistry of Materials, 2017. 29(1): p. 243-255.

4. Gorry, P.A., General least-squares smoothing and differentiation by the convolution (Savitzky-Golay) method. Analytical Chemistry, 1990. 62(6): p. 570-573.

5. Udvardi, B., et al., Effects of particle size on the attenuated total reflection spectrum of minerals. Applied Spectroscopy, 2017. 71(6): p. 1157-1168.

6. Bohren, C.F., et al., Absorption and scattering of light by small particles. 2008: John Wiley \& Sons.

7. Koike, C., et al., Effects of forsterite grain shape on infrared spectra. The Astrophysical Journal, 2010. 709(2): p. 983.

8. Kristova, P., et al., The effect of the particle size on the fundamental vibrations of the $\left[\mathrm{CO}_{3}{ }^{2-}\right]$ anion in calcite. The Journal of Physical Chemistry A, 2015. 119(20): p. 4891-4897.

9. $\quad$ Pabst, A., Structures of some tetragonal sheet silicates. Acta Crystallographica, 1959. 12: p. 733-739.

10. Mazzi, F., et al., Reexamination of cuprorivaite. American Mineralogist: Journal of Earth and Planetary Materials, 1962. 47: p. 409-411.

11. Errington, B., et al., Micronised Egyptian blue pigment: a novel near-infrared luminescent fingerprint dusting powder. Dyes and Pigments, 2016. 132: p. 310-315.

12. Weller, M., Inorganic materials chemistry: OUP primer 23. 1992: Oxford Univerity Press.

13. Lluveras, A., et al., Evidence for the use of Egyptian blue in an 11 th century mural altarprice by SEM-EDS, FTIR and SRXRD (church of Sant Pere, Terrassa, Spain). Archaeometry, 2010. 52(2): p. 308-319.

14. Mirti, P., et al., Spectrochemical and structural studies on a roman sample of Egyptian blue. Spectrochimica Acta Part A: Molecular and Biomolecular Spectroscopy, 1995. 51(3): p. 437-446.

15. Mahmoud, H.M., et al., Archaeometric analysis of pigments from the tomb of Kakbt-Djehuty (TT189), El-Qurna Necropolis, upper Egypt. ArcheoSciences, 2013(1): p. 19-33.

16. Bruni, S., et al., Spectrochemical characterization by micro-FTIR spectroscopy of blue pigments in different polychrome works of art. Vibrational Spectroscopy, 1999. 20(1): p. 15-25.

17. Tanvir, N.B., et al., Investigation of $\mathrm{CO}_{2}$ reaction with copper oxide nanoparticles for room temperature gas sensing. Journal of Materials Chemistry A, 2016. 4(14): p. 5294-5302. 
18. Azam, A., et al., Size-dependent antimicrobial properties of $\mathrm{CuO}$ nanoparticles against Gram-positive and-negative bacterial strains. International Journal of Nanomedicine, 2012. 7: p. 3527.

19. Zemnukhova, L.A., et al., Dependence of porosity of amorphous silicon dioxide prepared from rice straw on plant variety. BioResources, 2015. 10(2): p. 3713-3723.

20. Musić, S., et al., Precipitation of amorphous $\mathrm{SiO}_{2}$ particles and their properties. Brazilian Journal of Chemical Engineering, 2011. 28(1): p. 89-94.

21. Hofmeister, A., et al., Absorption and reflection infrared spectra of $M g O$ and other diatomic compounds. Monthly Notices of the Royal Astronomical Society, 2003. 345(1): p. 16-38.

22. Ahmed, M.K., et al., Fourier transform infrared and near-infrared spectroscopic methods for the detection of toxic diethylene glycol (DEG) contaminant in glycerin based cough syrup. Journal of Spectroscopy, 2010. 24(6): p. 601-608.

23. Choi, M.J., et al., An evaluation of nanostructured zinc oxide as a fluorescent powder for fingerprint detection. Journal of Materials Science, 2008. 43(2): p. 732-737.

24. Choi, M.J., et al., Metal-containing nanoparticles and nano-structured particles in fingermark detection. Forensic Science International, 2008. 179(2-3): p. 87-97.

25. Becue, A., et al., Detection of fingermarks by colloidal gold (MMD/SMD) - beyond the pH 3 limit. Forensic Science International, 2012. 219(1-3): p. 39-49.

26. Newland, T.G., et al., Further investigations into the single metal deposition (SMD II) technique for the detection of latent fingermarks. Forensic Science International, 2016. 268: p. 62-72. 